\title{
A proof of convergence for gradient descent in the training of artificial neural networks for constant target functions
}

\author{
Patrick Cheridito ${ }^{1}$, Arnulf Jentzen ${ }^{2,3}$, \\ Adrian Riekert ${ }^{4}$, and Florian Rossmannek ${ }^{5}$ \\ ${ }^{1}$ Department of Mathematics, ETH Zurich, \\ Zurich, Switzerland, e-mail: patrick.cheridito@math.ethz.ch \\ ${ }^{2}$ Faculty of Mathematics and Computer Science, University of Münster, \\ Münster, Germany, e-mail: ajentzen(a)uni-muenster.de \\ ${ }^{3}$ School of Data Science and Shenzhen Research Institute of Big Data, \\ The Chinese University of Hong Kong, Shenzhen, China, e-mail: ajentzen@cuhk.edu.cn \\ ${ }^{4}$ Faculty of Mathematics and Computer Science, University of Münster, \\ Münster, Germany, e-mail: ariekert(a)uni-muenster.de \\ ${ }^{5}$ Department of Mathematics, ETH Zurich, \\ Zurich, Switzerland, e-mail: florian.rossmannek@math.ethz.ch
}

February 22, 2021

\begin{abstract}
Gradient descent optimization algorithms are the standard ingredients that are used to train artificial neural networks (ANNs). Even though a huge number of numerical simulations indicate that gradient descent optimization methods do indeed convergence in the training of ANNs, until today there is no rigorous theoretical analysis which proves (or disproves) this conjecture. In particular, even in the case of the most basic variant of gradient descent optimization algorithms, the plain vanilla gradient descent method, it remains an open problem to prove or disprove the conjecture that gradient descent converges in the training of ANNs. In this article we solve this problem in the special situation where the target function under consideration is a constant function. More specifically, in the case of constant target functions we prove in the training of rectified fully-connected feedforward ANNs with one-hidden layer that the risk function of the gradient descent method does indeed converge to zero. Our mathematical analysis strongly exploits the property that the rectifier function is the activation function used in the considered ANNs. A key contribution of this work is to explicitly specify a Lyapunov function for the gradient flow system of the ANN parameters. This Lyapunov function is the central tool in our convergence proof of the gradient descent method.
\end{abstract}




\section{Contents}

1 Introduction $\quad 3$

2 Regularity properties of the risk functions and their gradients 5

2.1 Mathematical description of rectified artificial neural networks . . . . . . . 5

2.2 Smooth approximations of the rectifier function . . . . . . . . . . 5

2.3 Differentiability properties of the risk functions $\ldots \ldots \ldots \ldots$

2.4 Upper bounds for gradients of the risk functions . . . . . . . . . . . . 10

2.5 Properties of Lyapunov type functions . . . . . . . . . . . . . . . . . . 11

3 Convergence analysis for gradient flow processes 13

3.1 Deterministic Itô formulas for Lyapunov type functions $\ldots \ldots \ldots \ldots$

3.2 Deterministic Itô formulas for risk functions . . . . . . . . . . . . . . . . . 14

3.3 Convergence of the risks of gradient flow processes $\ldots \ldots \ldots \ldots$

4 Convergence analysis for gradient descent processes $\quad 16$

4.1 Lyapunov type estimates for gradient descent processes . . . . . . . . . . . 16

4.2 Convergence of the risks of gradient descent processes . . . . . . . . . . 18

4.3 Gradient descent processes with random initializations . . . . . . . . . . . 18

5 A priori estimates for general target functions 19 


\section{Introduction}

Gradient descent (GD) optimization schemes are the standard methods for the training of artificial neural networks (ANNs). Although a large number of numerical simulations hint that GD optimization methods do converge in the training of ANNs, in general there is no mathematical analysis in the scientific literature which proves (or disproves) the conjecture that GD optimization methods converge in the training of ANNs.

Even though the convergence of GD optimization methods is still an open problem of research, there are several promising approaches in the scientific literature which attack this problem. In particular, we refer, e.g., to [4, 5, 21] and the references mentioned therein for convergence results for GD optimization methods in the training of convex neural networks, we refer, e.g., to $[2,3,7,8,9,10,14,18,23,25,26]$ and the references mentioned therein for convergence results for GD optimization methods for the training of ANNs in the so-called overparametrized regime, we refer, e.g., to $[1,11,17,19]$ and the references mentioned therein for abstract convergence results for GD optimization methods which do not assume convexity of the considered objective functions, we refer, e.g., to [12, 13, 20, 24] and the references mentioned therein for results on the effect of initialization in the training of ANNs, and we refer, e.g., to $[6,16,20]$ and the references mentioned therein for lower bounds and divergence results for GD optimization methods. For more detailed overviews and further references on GD optimization schemes we also refer, e.g., to [22], [15, Section 1], and [11, Section 1.1].

A key idea of this work is to attack this challenging open problem of convergence of GD optimization methods in the training of ANNs in the situation of very special target functions: Our program is to first establish convergence in the case of constant target functions, thereafter, to prove convergence in the case of affine linear target functions, thereafter, to consider suitable continuous piecewise affine linear target functions, and, finally, to pass to the limit of general continuous target functions. In particular, the central contribution of this work is to solve this problem in the case of constant target functions. More formally, the main result of this article (see Theorem 4.4 in Subsection 4.2 below) proves that the risk function of the standard GD process converges to zero in the training of fully-connected rectified feedforward ANNs with one input, one output, and one hidden layer in the special situation where the target function under consideration is a constant function and where the input data is continuous uniformly distributed. In the next result, Theorem 1.1, we illustrate the findings of this work in more detail within this introductory section. Below Theorem 1.1 we add several explanatory comments regarding the statement of and the mathematical objects in Theorem 1.1 and we also highlight the key ideas of the proof of Theorem 1.1.

Theorem 1.1. Let $H \in \mathbb{N}, \alpha \in \mathbb{R}, \gamma \in(0, \infty)$, let $\|\cdot\|: \mathbb{R}^{3 H+1} \rightarrow[0, \infty)$ satisfy for all $\phi=$ $\left(\phi_{1}, \ldots, \phi_{3 H+1}\right) \in \mathbb{R}^{3 H+1}$ that $\|\phi\|=\left[\sum_{i=1}^{3 H+1}\left|\phi_{i}\right|^{2}\right]^{1 / 2}$, let $\sigma_{r}: \mathbb{R} \rightarrow \mathbb{R}, r \in[1, \infty]$, satisfy for all $r \in[1, \infty), x \in \mathbb{R}$ that $\sigma_{r}(x)=r^{-1} \ln \left(1+r^{-1} e^{r x}\right)$ and $\sigma_{\infty}(x)=\max \{x, 0\}$, let $\mathcal{N}_{r}=$ $\left(\mathcal{N}_{r}^{\phi}\right)_{\phi \in \mathbb{R}^{3 H+1}}: \mathbb{R}^{3 H+1} \rightarrow C(\mathbb{R}, \mathbb{R}), r \in[1, \infty]$, and $\mathcal{L}_{r}: \mathbb{R}^{3 H+1} \rightarrow \mathbb{R}, r \in[1, \infty]$, satisfy for all $r \in[1, \infty], \phi=\left(\phi_{1}, \ldots, \phi_{3 H+1}\right) \in \mathbb{R}^{3 H+1}, x \in \mathbb{R}$ that $\mathcal{N}_{r}^{\phi}(x)=\phi_{3 H+1}+\sum_{j=1}^{H} \phi_{2 H+j} \sigma_{r}\left(\phi_{j} x+\right.$ $\left.\phi_{H+j}\right)$ and $\mathcal{L}_{r}(\phi)=\int_{0}^{1}\left(\mathcal{N}_{r}^{\phi}(y)-\alpha\right)^{2} \mathrm{~d} y$, let $\mathcal{G}=\left(\mathcal{G}_{1}, \ldots, \mathcal{G}_{3 H+1}\right): \mathbb{R}^{3 H+1} \rightarrow \mathbb{R}^{3 H+1}$ satisfy for all $\phi \in\left\{\varphi \in \mathbb{R}^{3 H+1}:\left(\left(\nabla \mathcal{L}_{r}\right)(\varphi)\right)_{r \in \mathbb{N}}\right.$ is convergent $\}$ that $\mathcal{G}(\phi)=\lim _{r \rightarrow \infty}\left(\nabla \mathcal{L}_{r}\right)(\phi)$, and let $\Theta=\left(\Theta_{n}\right)_{n \in \mathbb{N}_{0}}: \mathbb{N}_{0} \rightarrow \mathbb{R}^{3 H+1}$ satisfy for all $n \in \mathbb{N}_{0}$ that $\Theta_{n+1}=\Theta_{n}-\gamma \mathcal{G}\left(\Theta_{n}\right)$ and $\gamma \leq$ $\left(4\left\|\Theta_{0}\right\|+6|\alpha|+2\right)^{-2}$. Then

(i) it holds for all $\phi \in\left\{\varphi \in \mathbb{R}^{3 H+1}: \mathcal{L}_{\infty}\right.$ is differentiable at $\left.\varphi\right\}$ that $\left(\nabla \mathcal{L}_{\infty}\right)(\phi)=\mathcal{G}(\phi)$,

(ii) it holds that $\sup _{n \in \mathbb{N}_{0}}\left\|\Theta_{n}\right\|<\infty$, and

(iii) it holds that $\lim \sup _{n \rightarrow \infty} \mathcal{L}_{\infty}\left(\Theta_{n}\right)=0$. 
Item (i) in Theorem 1.1 is a direct consequence of Corollary 2.9 below and items (ii) and (iii) in Theorem 1.1 are direct consequences of Corollary 4.5 below. Corollary 4.5, in turn, follows from Theorem 4.4, which is the main result of this article.

Let us next add a few comments regarding the mathematical objects appearing in Theorem 1.1. In Theorem 1.1 we study the training of ANNs with one input, one output, and one hidden layer. The natural number $H \in \mathbb{N}$ in Theorem 1.1 specifies the number of neurons on the hidden layer (the dimension of the hidden layer) in the ANN. Theorem 1.1 proves that the risk function of GD converges to zero in the special situation where the input data is continuous uniformly distributed and where the target function under consideration is a constant function. The real number $\alpha \in \mathbb{R}$ is precisely this constant with which the target function is assumed to coincide. The real number $\gamma \in(0, \infty)$ in Theorem 1.1 specifies the learning rate of the GD method.

In Theorem 1.1 we consider fully-connected feedforward ANNs with 1 neuron on the input layer, $H$ neurons on the hidden layer, and 1 neuron on the output layer. Therefore, the considered ANNs have precisely $2 H$ weights, $H+1$ biases, and $2 H+H+1=3 H+1$ ANN parameters overall. The function $\|\cdot\|: \mathbb{R}^{3 H+1} \rightarrow \mathbb{R}$ in Theorem 1.1 is nothing else but the standard norm on the space $\mathbb{R}^{3 H+1}$ of ANN parameters.

In Theorem 1.1 we study the training of ANNs with the rectifier function $\mathbb{R} \ni x \mapsto \sigma_{\infty}(x)=$ $\max \{x, 0\} \in \mathbb{R}$ as the activation function. Since the rectifier function $\sigma_{\infty}: \mathbb{R} \rightarrow \mathbb{R}$ in Theorem 1.1 is not differentiable at 0 , we have that the associated risk function also fails to be differentiable at some points in the ANN parameter space $\mathbb{R}^{3 H+1}$. In view of this, one needs to carefully choose the values for the driving gradient field in the GD optimization method at the points in the ANN parameter space $\mathbb{R}^{3 H+1}$ where the risk function is not differentiable. We accomplish this by approximating the rectifier function and the corresponding risk function through regularized versions of these functions. More formally, in Proposition 2.2 in Subsection 2.2 below we show that the functions $\sigma_{r}: \mathbb{R} \rightarrow \mathbb{R}, r \in[1, \infty]$, in Theorem 1.1 satisfy that for all $x \in \mathbb{R}, y \in \mathbb{R} \backslash\{0\}$ it holds that $\lim \sup _{r \rightarrow \infty}\left|\sigma_{r}(x)-\sigma_{\infty}(x)\right|=0$ and $\lim _{\sigma^{\prime}} \sup _{r \rightarrow \infty}\left|\left(\sigma_{r}\right)^{\prime}(y)-\left(\sigma_{\infty}\right)^{\prime}(y)\right|=0$. Observe that for all $r \in[1, \infty)$ it holds that $\sigma_{r} \in C^{\infty}(\mathbb{R}, \mathbb{R})$.

The functions $\mathcal{N}_{r}: \mathbb{R}^{3 H+1} \rightarrow C(\mathbb{R}, \mathbb{R}), r \in[1, \infty]$, in Theorem 1.1 describe the realization functions of the considered ANNs. More formally, note that for every $r \in[1, \infty]$ and every $\phi=\left(\phi_{1}, \ldots, \phi_{3 H+1}\right) \in \mathbb{R}^{3 H+1}$ we have that the function $\mathbb{R} \ni x \mapsto \mathcal{N}_{r}^{\phi}(x) \in \mathbb{R}$ is the realization function associated to the ANN with the activation function $\sigma_{r}: \mathbb{R} \rightarrow \mathbb{R}$ and the parameter vector $\phi=\left(\phi_{1}, \ldots, \phi_{3 H+1}\right)$. In particular, observe that for every ANN parameter vector $\phi \in$ $\mathbb{R}^{3 H+1}$ we have that $\mathbb{R} \ni x \mapsto \mathcal{N}_{\infty}^{\phi}(x) \in \mathbb{R}$ is the realization function associated to the rectified ANN with the parameter vector $\phi$.

The process $\Theta=\left(\Theta_{n}\right)_{n \in \mathbb{N}_{0}}: \mathbb{N}_{0} \rightarrow \mathbb{R}^{3 H+1}$ in Theorem 1.1 is the GD process with constant learning rate $\gamma$. Note that the learning rate $\gamma$ in Theorem 1.1 is assumed to be sufficiently small in the sense that $\gamma \leq\left(4\left\|\Theta_{0}\right\|+6|\alpha|+2\right)^{-2}$. Under this assumption, Theorem 1.1 reveals that the risk of the GD process $\mathcal{L}_{\infty}\left(\Theta_{n}\right), n \in \mathbb{N}_{0}$, does indeed converge to zero as the number of GD steps $n$ increases to infinity.

Let us also add a few comments on the proof of Theorem 1.1. A key new observation of this article is the fact that in the situation of Theorem 1.1 we have that the function

$$
\mathbb{R}^{3 H+1} \ni\left(\phi_{1}, \ldots, \phi_{3 H+1}\right) \mapsto\left(\sum_{i=1}^{3 H+1}\left|\phi_{i}\right|^{2}\right)+\left(\phi_{3 H+1}-2 \alpha\right)^{2} \in \mathbb{R}
$$

is a Lyapunov function for the gradient flow system of the ANN parameters. We refer to item (iii) in Proposition 2.14 in Subsection 2.5 and Lemma 3.2 in Subsection 3.1 for the proof of this statement. In addition, in Lemma 4.3 in Subsection 4.1 we show that the function in (1) is also a Lyapunov function for the time-discrete GD processes if the learning rate is sufficiently small. We also would like to emphasize that the term $\left(\phi_{3 H+1}-2 \alpha\right)^{2}$ in (1) is essential for the function in (1) to serve as a Lyapunov function. In particular, we would like to point out that 
the function $\mathbb{R}^{3 H+1} \ni \phi \mapsto\|\phi\|^{2} \in \mathbb{R}$ fails to be a Lyapunov function for the gradient flow system of the ANN parameters.

The remainder of this article is structured as follows. In Section 2 we present the mathematical framework which we use to study the considered GD processes and we also establish several regularity properties for the considered risk functions and their gradients. In Section 3 we use the findings from Section 2 to establish that the risks of the considered time-continuous gradient flow processes converge to zero. In Section 4 we prove that the risks of the considered time-discrete GD processes converge to zero. The key ingredient in our convergence proofs for gradient flow and GD processes in Sections 3 and 4 are suitable a priori estimates (which we achieve by means of the Lyapunov function in (1) above) for the gradient flow processes (see Lemma 3.2 in Subsection 3.1) and the GD processes (see Lemma 4.3 in Subsection 4.1). In Section 5 we derive - to stimulate further research activities - related a priori bounds in the case of general target functions.

\section{Regularity properties of the risk functions and their gradients}

In Section 2 we present in Setting 2.1 the mathematical framework which we use to study the considered GD processes and we also establish several regularity results for the considered risk functions and their gradients. Most notably, we establish in Propositions 2.12 and 2.14 in Subsection 2.5 below that the gradient flow system for the ANN parameters in Setting 2.1 admits an appropriate Lyapunov function. In particular, in item (iii) in Proposition 2.14 we prove that the function $V: \mathbb{R}^{3 H+1} \rightarrow \mathbb{R}$ in Setting 2.1 serves as a Lyapunov function.

We also note that the results in Proposition 2.2 in Subsection 2.2, in Lemma 2.4 in Subsection 2.3, and in Corollary 2.5 in Subsection 2.3 are all well-known in the literature and we include in this section detailed proofs for Proposition 2.2, Lemma 2.4, and Corollary 2.5 only for completeness.

\subsection{Mathematical description of rectified artificial neural networks}

Setting 2.1. Let $H \in \mathbb{N}, \alpha \in \mathbb{R}$, let $\mathfrak{w}=\left(\left(\mathfrak{w}_{1}^{\phi}, \ldots, \mathfrak{w}_{H}^{\phi}\right)\right)_{\phi \in \mathbb{R}^{3 H+1}}: \mathbb{R}^{3 H+1} \rightarrow \mathbb{R}^{H}, \mathfrak{b}=$ $\left(\left(\mathfrak{b}_{1}^{\phi}, \ldots, \mathfrak{b}_{H}^{\phi}\right)\right)_{\phi \in \mathbb{R}^{3 H+1}}: \mathbb{R}^{3 H+1} \rightarrow \mathbb{R}^{H}, \mathfrak{v}=\left(\left(\mathfrak{v}_{1}^{\phi}, \ldots, \mathfrak{v}_{H}^{\phi}\right)\right)_{\phi \in \mathbb{R}^{3 H+1}}: \mathbb{R}^{3 H+1} \rightarrow \mathbb{R}^{H}$, and $\mathfrak{c}=$ $\left(\mathfrak{c}^{\phi}\right)_{\phi \in \mathbb{R}^{3 H+1}}: \mathbb{R}^{3 H+1} \rightarrow \mathbb{R}$ satisfy for all $\phi=\left(\phi_{1}, \ldots, \phi_{3 H+1}\right) \in \mathbb{R}^{3 H+1}, j \in\{1,2, \ldots, H\}$ that $\mathfrak{w}_{j}^{\phi}=\phi_{j}, \mathfrak{b}_{j}^{\phi}=\phi_{H+j}, \mathfrak{v}_{j}^{\phi}=\phi_{2 H+j}$, and $\mathfrak{c}^{\phi}=\phi_{3 H+1}$, let $\sigma_{r}: \mathbb{R} \rightarrow \mathbb{R}, r \in[1, \infty]$, satisfy for all $r \in[1, \infty), x \in \mathbb{R}$ that $\sigma_{r}(x)=r^{-1} \ln \left(1+r^{-1} e^{r x}\right)$ and $\sigma_{\infty}(x)=\max \{x, 0\}$, let $\mathcal{N}_{r}=\left(\mathcal{N}_{r}^{\phi}\right)_{\phi \in \mathbb{R}^{3 H+1}}: \mathbb{R}^{3 H+1} \rightarrow C(\mathbb{R}, \mathbb{R}), r \in[1, \infty]$, and $\mathcal{L}_{r}: \mathbb{R}^{3 H+1} \rightarrow \mathbb{R}, r \in[1, \infty]$, satisfy for all $r \in[1, \infty], \phi \in \mathbb{R}^{3 H+1}, x \in \mathbb{R}$ that $\mathcal{N}_{r}^{\phi}(x)=\mathfrak{c}^{\phi}+\sum_{j=1}^{H} \mathfrak{v}_{j}^{\phi} \sigma_{r}\left(\mathfrak{w}_{j}^{\phi} x+\mathfrak{b}_{j}^{\phi}\right)$ and $\mathcal{L}_{r}(\phi)=\int_{0}^{1}\left(\mathcal{N}_{r}^{\phi}(y)-\alpha\right)^{2} \mathrm{~d} y$, let $\mathcal{G}=\left(\mathcal{G}_{1}, \ldots, \mathcal{G}_{3 H+1}\right): \mathbb{R}^{3 H+1} \rightarrow \mathbb{R}^{3 H+1}$ satisfy for all $\phi \in\{\varphi \in$ $\mathbb{R}^{3 H+1}:\left(\left(\nabla \mathcal{L}_{r}\right)(\varphi)\right)_{r \in \mathbb{N}}$ is convergent $\}$ that $\mathcal{G}(\phi)=\lim _{r \rightarrow \infty}\left(\nabla \mathcal{L}_{r}\right)(\phi)$, let $\|\cdot\|:\left(\bigcup_{n \in \mathbb{N}} \mathbb{R}^{n}\right) \rightarrow$ $[0, \infty)$ and $\langle\cdot, \cdot\rangle:\left(\bigcup_{n \in \mathbb{N}}\left(\mathbb{R}^{n} \times \mathbb{R}^{n}\right)\right) \rightarrow \mathbb{R}$ satisfy for all $n \in \mathbb{N}, x=\left(x_{1}, \ldots, x_{n}\right), y=\left(y_{1}, \ldots, y_{n}\right) \in$ $\mathbb{R}^{n}$ that $\|x\|=\left[\sum_{i=1}^{n}\left|x_{i}\right|^{2}\right]^{1 / 2}$ and $\langle x, y\rangle=\sum_{i=1}^{n} x_{i} y_{i}$, and let $I_{j}^{\phi} \subseteq \mathbb{R}, \phi \in \mathbb{R}^{3 H+1}, j \in$ $\{1,2, \ldots, H\}$, and $V: \mathbb{R}^{3 H+1} \rightarrow \mathbb{R}$ satisfy for all $\phi \in \mathbb{R}^{3 H+1}, j \in\{1,2, \ldots, H\}$ that $I_{j}^{\phi}=$ $\left\{x \in[0,1]: \mathfrak{w}_{j}^{\phi} x+\mathfrak{b}_{j}^{\phi}>0\right\}$ and $V(\phi)=\|\phi\|^{2}+\left(\mathfrak{c}^{\phi}-2 \alpha\right)^{2}$.

\subsection{Smooth approximations of the rectifier function}

Proposition 2.2. Let $\sigma_{r}: \mathbb{R} \rightarrow \mathbb{R}, r \in[1, \infty]$, satisfy for all $r \in[1, \infty), x \in \mathbb{R}$ that $\sigma_{r}(x)=$ $r^{-1} \ln \left(1+r^{-1} e^{r x}\right)$ and $\sigma_{\infty}(x)=\max \{x, 0\}$. Then

(i) it holds for all $r \in[1, \infty)$ that $\sigma_{r} \in C^{\infty}(\mathbb{R}, \mathbb{R})$,

(ii) it holds for all $r \in[1, \infty), x \in \mathbb{R}$ that $0<\sigma_{r}(x)<\sigma_{\infty}(x)+1$, 
(iii) it holds for all $x \in \mathbb{R}$ that $\lim \sup _{r \rightarrow \infty}\left|\sigma_{r}(x)-\sigma_{\infty}(x)\right|=0$,

(iv) it holds for all $r \in[1, \infty), x \in \mathbb{R}$ that $0<\left(\sigma_{r}\right)^{\prime}(x)<1$, and

(v) it holds for all $x \in \mathbb{R}$ that $\lim \sup _{r \rightarrow \infty}\left|\left(\sigma_{r}\right)^{\prime}(x)-\mathbb{1}_{(0, \infty)}(x)\right|=0$.

Proof of Proposition 2.2. Observe that the fact that $\left(\mathbb{R} \ni x \mapsto e^{x} \in \mathbb{R}\right) \in C^{\infty}(\mathbb{R}, \mathbb{R})$, the fact that $((0, \infty) \ni x \mapsto \ln (x) \in \mathbb{R}) \in C^{\infty}((0, \infty), \mathbb{R})$, and the chain rule prove item (i). Next note that for all $r \in[1, \infty), x \in(-\infty, 0]$ it holds that $1<1+r^{-1} e^{r x} \leq 2$ and therefore

$$
0<\sigma_{r}(x) \leq r^{-1} \ln (2)<r^{-1} \leq 1=\sigma_{\infty}(x)+1 .
$$

This establishes for all $x \in(-\infty, 0]$ that $\lim _{\sup _{r \rightarrow \infty}}\left|\sigma_{r}(x)-\sigma_{\infty}(x)\right| \leq \limsup _{r \rightarrow \infty}\left(r^{-1}\right)=0$. Moreover, observe that for all $r \in[1, \infty), x \in(0, \infty)$ it holds that

$$
0=r^{-1} \ln (1)<\sigma_{r}(x) \leq r^{-1} \ln \left(2 e^{r x}\right)=x+r^{-1} \ln (2)<x+1=\sigma_{\infty}(x)+1 .
$$

This and (2) prove item (ii). In addition, note that for all $r \in[1, \infty), x \in(0, \infty)$ it holds that $\sigma_{r}(x) \geq r^{-1} \ln \left(r^{-1} e^{r x}\right)=x-r^{-1} \ln (r)$. Combining this with (3) demonstrates for all $x \in(0, \infty)$ that

$$
\begin{aligned}
\limsup _{r \rightarrow \infty}\left|\sigma_{r}(x)-\sigma_{\infty}(x)\right| & =\limsup _{r \rightarrow \infty}\left|\sigma_{r}(x)-x\right| \\
& \leq \limsup _{r \rightarrow \infty}\left[\max \left\{r^{-1} \ln (2), r^{-1} \ln (r)\right\}\right]=0,
\end{aligned}
$$

which completes the proof of item (iii). To prove item (iv), observe that the chain rule implies for all $r \in[1, \infty), x \in \mathbb{R}$ that

$$
\left(\sigma_{r}\right)^{\prime}(x)=\frac{1}{r}\left[\frac{e^{r x}}{1+r^{-1} e^{r x}}\right]=\frac{1}{1+r e^{-r x}} .
$$

This demonstrates for all $r \in[1, \infty), x \in \mathbb{R}$ that $0<\left(\sigma_{r}\right)^{\prime}(x)<1$, which establishes item (iv). Next note that (5) and the fact that for all $r \in[1, \infty), x \in(-\infty, 0]$ it holds that $e^{-r x} \geq 1$ show that for all $r \in[1, \infty), x \in(-\infty, 0]$ it holds that $\left(\sigma_{r}\right)^{\prime}(x) \leq \frac{1}{1+r}$. On the other hand, observe that for all $x \in(0, \infty)$ we have that $\lim _{r \rightarrow \infty}\left(r e^{-r x}\right)=0$ and thus $\lim _{r \rightarrow \infty}\left(\sigma_{r}\right)^{\prime}(x)=1$. This establishes item (v). The proof of Proposition 2.2 is thus complete.

\subsection{Differentiability properties of the risk functions}

Proposition 2.3. Assume Setting 2.1 and let $\phi=\left(w_{1}, \ldots, w_{H}, b_{1}, \ldots, b_{H}, v_{1}, \ldots, v_{H}, c\right) \in$ $\mathbb{R}^{3 H+1}$. Then

(i) it holds for all $r \in[1, \infty)$ that $\mathcal{L}_{r} \in C^{1}\left(\mathbb{R}^{3 H+1}, \mathbb{R}\right)$,

(ii) it holds for all $r \in[1, \infty), j \in\{1,2, \ldots, H\}$ that

$$
\begin{aligned}
& \left(\frac{\partial}{\partial w_{j}} \mathcal{L}_{r}\right)(\phi)=2 v_{j} \int_{0}^{1} x\left[\left(\sigma_{r}\right)^{\prime}\left(w_{j} x+b_{j}\right)\right]\left(\mathcal{N}_{r}^{\phi}(x)-\alpha\right) \mathrm{d} x \\
& \left(\frac{\partial}{\partial b_{j}} \mathcal{L}_{r}\right)(\phi)=2 v_{j} \int_{0}^{1}\left[\left(\sigma_{r}\right)^{\prime}\left(w_{j} x+b_{j}\right)\right]\left(\mathcal{N}_{r}^{\phi}(x)-\alpha\right) \mathrm{d} x \\
& \left(\frac{\partial}{\partial v_{j}} \mathcal{L}_{r}\right)(\phi)=2 \int_{0}^{1}\left[\sigma_{r}\left(w_{j} x+b_{j}\right)\right]\left(\mathcal{N}_{r}^{\phi}(x)-\alpha\right) \mathrm{d} x \\
& \left(\frac{\partial}{\partial c} \mathcal{L}_{r}\right)(\phi)=2 \int_{0}^{1}\left(\mathcal{N}_{r}^{\phi}(x)-\alpha\right) \mathrm{d} x
\end{aligned}
$$

(iii) it holds that $\lim \sup _{r \rightarrow \infty}\left|\mathcal{L}_{r}(\phi)-\mathcal{L}_{\infty}(\phi)\right|=0$, 
(iv) it holds that $\lim \sup _{r \rightarrow \infty}\left\|\left(\nabla \mathcal{L}_{r}\right)(\phi)-\mathcal{G}(\phi)\right\|=0$, and

(v) it holds for all $j \in\{1,2, \ldots, H\}$ that

$$
\begin{aligned}
\mathcal{G}_{j}(\phi) & =2 v_{j} \int_{I_{j}^{\phi}} x\left(\mathcal{N}_{\infty}^{\phi}(x)-\alpha\right) \mathrm{d} x, \\
\mathcal{G}_{H+j}(\phi) & =2 v_{j} \int_{I_{j}^{\phi}}\left(\mathcal{N}_{\infty}^{\phi}(x)-\alpha\right) \mathrm{d} x \\
\mathcal{G}_{2 H+j}(\phi) & =2 \int_{0}^{1}\left[\sigma_{\infty}\left(w_{j} x+b_{j}\right)\right]\left(\mathcal{N}_{\infty}^{\phi}(x)-\alpha\right) \mathrm{d} x, \\
\mathcal{G}_{3 H+1}(\phi) & =2 \int_{0}^{1}\left(\mathcal{N}_{\infty}^{\phi}(x)-\alpha\right) \mathrm{d} x .
\end{aligned}
$$

Proof of Proposition 2.3. Note that Proposition 2.2, the chain rule, and the dominated convergence theorem establish items (i) and (ii). Next observe that Proposition 2.2 demonstrates for all $x \in[0,1]$ that $\lim _{r \rightarrow \infty}\left(\mathcal{N}_{r}^{\phi}(x)-\alpha\right)=\mathcal{N}_{\infty}^{\phi}(x)-\alpha$. Furthermore, note that Proposition 2.2 shows that for all $x \in[0,1], r \in[1, \infty)$ it holds that

$$
\begin{aligned}
\left|\mathcal{N}_{r}^{\phi}(x)-\alpha\right| & \leq|\alpha|+|c|+\sum_{j=1}^{H}\left|v_{j}\right|\left(\sigma_{\infty}\left(w_{j} x+b_{j}\right)+1\right) \\
& \leq|\alpha|+|c|+\sum_{j=1}^{H}\left|v_{j}\right|\left(\left|w_{j}\right|+\left|b_{j}\right|+1\right) .
\end{aligned}
$$

The dominated convergence theorem hence proves that $\lim _{r \rightarrow \infty} \mathcal{L}_{r}(\phi)=\mathcal{L}_{\infty}(\phi)$, which establishes item (iii). Moreover, observe that the fact that $\forall x \in[0,1]: \lim _{r \rightarrow \infty}\left(\mathcal{N}_{r}^{\phi}(x)-\alpha\right)=$ $\mathcal{N}_{\infty}^{\phi}(x)-\alpha,(8)$, and the dominated convergence theorem prove that

$$
\lim _{r \rightarrow \infty}\left[\left(\frac{\partial}{\partial c} \mathcal{L}_{r}\right)(\phi)\right]=2 \int_{0}^{1}\left(\mathcal{N}_{\infty}^{\phi}(x)-\alpha\right) \mathrm{d} x .
$$

Next note that Proposition 2.2 shows for all $x \in[0,1], j \in\{1,2, \ldots, H\}$ that

$$
\begin{aligned}
\lim _{r \rightarrow \infty}\left[x\left[\left(\sigma_{r}\right)^{\prime}\left(w_{j} x+b_{j}\right)\right]\left(\mathcal{N}_{r}^{\phi}(x)-\alpha\right)\right] & =x\left(\mathcal{N}_{\infty}^{\phi}(x)-\alpha\right) \mathbb{1}_{(0, \infty)}\left(w_{j} x+b_{j}\right) \\
& =x\left(\mathcal{N}_{\infty}^{\phi}(x)-\alpha\right) \mathbb{1}_{I_{j}^{\phi}}(x)
\end{aligned}
$$

and

$$
\begin{aligned}
\lim _{r \rightarrow \infty}\left[\left[\left(\sigma_{r}\right)^{\prime}\left(w_{j} x+b_{j}\right)\right]\left(\mathcal{N}_{r}^{\phi}(x)-\alpha\right)\right] & =\left(\mathcal{N}_{\infty}^{\phi}(x)-\alpha\right) \mathbb{1}_{(0, \infty)}\left(w_{j} x+b_{j}\right) \\
& =\left(\mathcal{N}_{\infty}^{\phi}(x)-\alpha\right) \mathbb{1}_{I_{j}^{\phi}}(x) .
\end{aligned}
$$

Furthermore, observe that Proposition 2.2 and (8) prove that for all $r \in[1, \infty), x \in[0,1]$, $j \in\{1,2, \ldots, H\}$ it holds that

$$
\begin{aligned}
& \left|x\left[\left(\sigma_{r}\right)^{\prime}\left(w_{j} x+b_{j}\right)\right]\left(\mathcal{N}_{r}^{\phi}(x)-\alpha\right)\right| \\
& \leq\left|\left[\left(\sigma_{r}\right)^{\prime}\left(w_{j} x+b_{j}\right)\right]\left(\mathcal{N}_{r}^{\phi}(x)-\alpha\right)\right| \\
& \leq\left|\mathcal{N}_{r}^{\phi}(x)-\alpha\right| \leq|\alpha|+|c|+\sum_{j=1}^{H}\left|v_{j}\right|\left(\left|w_{j}\right|+\left|b_{j}\right|+1\right) .
\end{aligned}
$$

The dominated convergence theorem hence proves for all $j \in\{1,2, \ldots, H\}$ that

$$
\lim _{r \rightarrow \infty}\left[\left(\frac{\partial}{\partial w_{j}} \mathcal{L}_{r}\right)(\phi)\right]=2 v_{j} \int_{0}^{1} x\left(\mathcal{N}_{\infty}^{\phi}(x)-\alpha\right) \mathbb{1}_{I_{j}^{\phi}}(x) \mathrm{d} x=2 v_{j} \int_{I_{j}^{\phi}} x\left(\mathcal{N}_{\infty}^{\phi}(x)-\alpha\right) \mathrm{d} x
$$

and

$$
\lim _{r \rightarrow \infty}\left[\left(\frac{\partial}{\partial b_{j}} \mathcal{L}_{r}\right)(\phi)\right]=2 v_{j} \int_{0}^{1}\left(\mathcal{N}_{\infty}^{\phi}(x)-\alpha\right) \mathbb{1}_{I_{j}^{\phi}}(x) \mathrm{d} x=2 v_{j} \int_{I_{j}^{\phi}}\left(\mathcal{N}_{\infty}^{\phi}(x)-\alpha\right) \mathrm{d} x .
$$


Moreover, note that Proposition 2.2 and (8) show that for all $r \in[1, \infty), x \in[0,1], j \in$ $\{1,2, \ldots, H\}$ it holds that

$$
\lim _{r \rightarrow \infty}\left[\left[\sigma_{r}\left(w_{j} x+b_{j}\right)\right]\left(\mathcal{N}_{r}^{\phi}(x)-\alpha\right)\right]=\left[\sigma_{\infty}\left(w_{j} x+b_{j}\right)\right]\left(\mathcal{N}_{\infty}^{\phi}(x)-\alpha\right)
$$

and

$$
\begin{aligned}
& \left|\left[\sigma_{r}\left(w_{j} x+b_{j}\right)\right]\left(\mathcal{N}_{r}^{\phi}(x)-\alpha\right)\right| \\
& \leq\left(\sigma_{\infty}\left(w_{j} x+b_{j}\right)+1\right)\left|\mathcal{N}_{r}^{\phi}(x)-\alpha\right| \\
& \leq\left(1+\left|w_{j}\right|+\left|b_{j}\right|\right)\left|\mathcal{N}_{r}^{\phi}(x)-\alpha\right| \\
& \leq\left(1+\left|w_{j}\right|+\left|b_{j}\right|\right)\left(|\alpha|+|c|+\sum_{j=1}^{H}\left|v_{j}\right|\left(\left|w_{j}\right|+\left|b_{j}\right|+1\right)\right) .
\end{aligned}
$$

This and the dominated convergence theorem demonstrate for all $j \in\{1,2, \ldots, H\}$ that

$$
\lim _{r \rightarrow \infty}\left[\left(\frac{\partial}{\partial v_{j}} \mathcal{L}_{r}\right)(\phi)\right]=2 \int_{0}^{1}\left[\sigma_{\infty}\left(w_{j} x+b_{j}\right)\right]\left(\mathcal{N}_{\infty}^{\phi}(x)-\alpha\right) \mathrm{d} x .
$$

Combining this, (9), (13), and (14) establishes items (iv) and (v). The proof of Proposition 2.3 is thus complete.

Lemma 2.4. Let $\mathfrak{u} \in \mathbb{R}, \mathfrak{v} \in(\mathfrak{u}, \infty)$, let $f: \mathbb{R} \times[\mathfrak{u}, \mathfrak{v}] \rightarrow \mathbb{R}$ be locally Lipschitz continuous, let $F: \mathbb{R} \rightarrow \mathbb{R}$ satisfy for all $x \in \mathbb{R}$ that

$$
F(x)=\int_{\mathfrak{u}}^{\mathfrak{v}} f(x, s) \mathrm{d} s,
$$

let $x \in \mathbb{R}$, let $E \subseteq[\mathfrak{u}, \mathfrak{v}]$ be measurable, assume $\int_{[\mathfrak{u}, \mathfrak{v}] \backslash E} 1 \mathrm{~d} s=0$, and assume for all $s \in E$ that $\mathbb{R} \ni v \mapsto f(v, s) \in \mathbb{R}$ is differentiable at $x$. Then

(i) it holds that $F$ is differentiable at $x$ and

(ii) it holds that

$$
F^{\prime}(x)=\int_{E}\left(\frac{\partial}{\partial x} f\right)(x, s) \mathrm{d} s .
$$

Proof of Lemma 2.4. Observe that the assumption that $\int_{[\mathfrak{u}, \mathfrak{v}] \backslash E} 1 \mathrm{~d} s=0$ ensures that for all $h \in \mathbb{R} \backslash\{0\}$ we have that

$$
h^{-1}[F(x+h)-F(x)]=\int_{\mathfrak{u}}^{\mathfrak{v}} h^{-1}[f(x+h, s)-f(x, s)] \mathrm{d} s=\int_{E} h^{-1}[f(x+h, s)-f(x, s)] \mathrm{d} s .
$$

Next note that the assumption that for all $s \in E$ it holds that $\mathbb{R} \ni v \mapsto f(v, s) \in \mathbb{R}$ is differentiable at $x$ implies that for all $s \in E$ it holds that

$$
\lim _{|h| \searrow 0}\left(h^{-1}[f(x+h, s)-f(x, s)]\right)=\left(\frac{\partial}{\partial x} f\right)(x, s) .
$$

Furthermore, observe that the assumption that $f$ is locally Lipschitz continuous ensures that for all $\delta \in(0, \infty)$ there exists $C \in(0, \infty)$ such that for all $h \in[-\delta, \delta] \backslash\{0\}, s \in[\mathfrak{u}, \mathfrak{v}]$ we have that $\left|h^{-1}[f(x+h, s)-f(x, s)]\right| \leq C$. Combining this, (20), (21), and the dominated convergence theorem establishes that

$$
\begin{aligned}
\lim _{|h| \searrow 0}\left(h^{-1}[F(x+h)-F(x)]\right) & =\int_{E}\left[\lim _{|h| \searrow 0}\left(h^{-1}[f(x+h, s)-f(x, s)]\right)\right] \mathrm{d} s \\
& =\int_{E}\left(\frac{\partial}{\partial x} f\right)(x, s) \mathrm{d} s .
\end{aligned}
$$

This completes the proof of Lemma 2.4. 
Corollary 2.5. Let $n \in \mathbb{N}, j \in\{1,2, \ldots, n\}, \mathfrak{u} \in \mathbb{R}, \mathfrak{v} \in(\mathfrak{u}, \infty)$, let $f: \mathbb{R}^{n} \times[\mathfrak{u}, \mathfrak{v}] \rightarrow \mathbb{R}$ be locally Lipschitz continuous, let $F: \mathbb{R}^{n} \rightarrow \mathbb{R}$ satisfy for all $x \in \mathbb{R}^{n}$ that

$$
F(x)=\int_{\mathfrak{u}}^{\mathfrak{v}} f(x, s) \mathrm{d} s,
$$

let $x_{1}, x_{2}, \ldots, x_{n} \in \mathbb{R}$, let $E \subseteq[\mathfrak{u}, \mathfrak{v}]$ be measurable, assume $\int_{[\mathfrak{u}, \mathfrak{v}] \backslash E} 1 \mathrm{~d} s=0$, and assume for all $s \in E$ that $\mathbb{R} \ni v \mapsto f\left(x_{1}, \ldots, x_{j-1}, v, x_{j+1}, \ldots, x_{n}, s\right) \in \mathbb{R}$ is differentiable at $x_{j}$. Then

(i) it holds that $\mathbb{R} \ni v \mapsto F\left(x_{1}, \ldots, x_{j-1}, v, x_{j+1}, \ldots, x_{n}\right) \in \mathbb{R}$ is differentiable at $x_{j}$ and

(ii) it holds that

$$
\left(\frac{\partial}{\partial x_{j}} F\right)\left(x_{1}, \ldots, x_{n}\right)=\int_{E}\left(\frac{\partial}{\partial x_{j}} f\right)\left(x_{1}, \ldots, x_{n}, s\right) \mathrm{d} s .
$$

Proof of Corollary 2.5. Note that Lemma 2.4 establishes items (i) and (ii). The proof of Corollary 2.5 is thus complete.

Lemma 2.6. Assume Setting 2.1 and let $\phi=\left(\phi_{1}, \ldots, \phi_{3 H+1}\right) \in \mathbb{R}^{3 H+1}$. Then

(i) it holds for all $j \in \mathbb{N} \cap(2 H, 3 H+1]$ that $\mathbb{R} \ni v \mapsto \mathcal{L}_{\infty}\left(\phi_{1}, \ldots, \phi_{j-1}, v, \phi_{j+1}, \ldots, \phi_{3 H+1}\right) \in \mathbb{R}$ is differentiable at $\phi_{j}$ and

(ii) it holds for all $j \in \mathbb{N} \cap(2 H, 3 H+1]$ that $\left(\frac{\partial}{\partial \phi_{j}} \mathcal{L}_{\infty}\right)(\phi)=\mathcal{G}_{j}(\phi)$.

Proof of Lemma 2.6. Observe that the fact that $\sigma_{\infty}$ is Lipschitz continuous assures that

$$
\mathbb{R}^{H+1} \times[0,1] \ni\left(u_{1}, \ldots, u_{H+1}, x\right) \mapsto\left(\mathcal{N}_{\infty}^{\left(\phi_{1}, \ldots, \phi_{2 H}, u_{1}, \ldots, u_{H+1}\right)}(x)-\alpha\right)^{2} \in \mathbb{R}
$$

is locally Lipschitz continuous. In addition, note that for all $u_{1}, u_{2}, \ldots, u_{H+1} \in \mathbb{R}, j \in$ $\{1,2, \ldots, H+1\}, x \in[0,1]$ it holds that

$$
\mathbb{R} \ni v \mapsto\left(\mathcal{N}_{\infty}^{\left(\phi_{1}, \ldots, \phi_{2 H}, u_{1}, \ldots, u_{j-1}, v, u_{j+1}, \ldots, u_{H+1}\right)}(x)-\alpha\right)^{2} \in \mathbb{R}
$$

is differentiable at $u_{j}$. Moreover, observe that the chain rule implies that for all $j \in\{1,2, \ldots, H\}$, $x \in[0,1]$ it holds that

$$
\frac{\partial}{\partial \phi_{2 H+j}}\left[\left(\mathcal{N}_{\infty}^{\phi}(x)-\alpha\right)^{2}\right]=2\left[\sigma_{\infty}\left(\phi_{j} x+\phi_{H+j}\right)\right]\left(\mathcal{N}_{\infty}^{\phi}(x)-\alpha\right)
$$

and

$$
\frac{\partial}{\partial \phi_{3 H+1}}\left[\left(\mathcal{N}_{\infty}^{\phi}(x)-\alpha\right)^{2}\right]=2\left(\mathcal{N}_{\infty}^{\phi}(x)-\alpha\right) .
$$

Combining this, Corollary 2.5, and (7) establishes items (i) and (ii). The proof of Lemma 2.6 is thus complete.

Lemma 2.7. Assume Setting 2.1, let $\phi=\left(\phi_{1}, \ldots, \phi_{3 H+1}\right) \in \mathbb{R}^{3 H+1}$, and let $j \in\{1,2, \ldots, H\}$, $i \in\{j, H+j\}$ satisfy $\left|\phi_{j}\right|+\left|\phi_{H+j}\right|>0$. Then

(i) it holds that $\mathbb{R} \ni v \mapsto \mathcal{L}_{\infty}\left(\phi_{1}, \ldots, \phi_{i-1}, v, \phi_{i+1}, \ldots, \phi_{3 H+1}\right) \in \mathbb{R}$ is differentiable at $\phi_{i}$ and

(ii) it holds that $\left(\frac{\partial}{\partial \phi_{i}} \mathcal{L}_{\infty}\right)(\phi)=\mathcal{G}_{i}(\phi)$.

Proof of Lemma 2.7. Throughout this proof let $E \subseteq \mathbb{R}$ satisfy $E=\left\{x \in[0,1]: \phi_{j} x+\phi_{H+j} \neq 0\right\}$. Note that the assumption that $\left|\phi_{j}\right|+\left|\phi_{H+j}\right|>0$ implies that $\#([0,1] \backslash E) \leq 1$. This shows that $\int_{[0,1] \backslash E} 1 \mathrm{~d} s=0$. Next observe that the fact that $\sigma_{\infty}$ is Lipschitz continuous ensures that

$$
\mathbb{R}^{2 H} \times[0,1] \ni\left(u_{1}, \ldots, u_{2 H}, x\right) \mapsto\left(\mathcal{N}_{\infty}^{\left(u_{1}, \ldots, u_{2 H}, \phi_{2 H+1}, \ldots, \phi_{3 H+1}\right)}(x)-\alpha\right)^{2} \in \mathbb{R}
$$


is locally Lipschitz continuous. In addition, note that for all $x \in \mathbb{R} \backslash\{0\}$ it holds that $\sigma_{\infty}$ is differentiable at $x$. Furthermore, observe that for all $x \in \mathbb{R} \backslash\{0\}$ it holds that $\left(\sigma_{\infty}\right)^{\prime}(x)=$ $\mathbb{1}_{(0, \infty)}(x)$. This and the chain rule prove for all $x \in E$ that

$$
\mathbb{R} \ni v \mapsto\left(\mathcal{N}_{\infty}^{\left(\phi_{1}, \ldots, \phi_{j-1}, v, \phi_{j+1}, \ldots, \phi_{3 H+1}\right)}(x)-\alpha\right)^{2} \in \mathbb{R}
$$

is differentiable at $\phi_{j}$ and

$$
\frac{\partial}{\partial \phi_{j}}\left(\mathcal{N}_{\infty}^{\phi}(x)-\alpha\right)^{2}=2 \phi_{2 H+j} x\left(\mathcal{N}_{\infty}^{\phi}(x)-\alpha\right) \mathbb{1}_{(0, \infty)}\left(\phi_{j} x+\phi_{H+j}\right)=2 \phi_{2 H+j} x\left(\mathcal{N}_{\infty}^{\phi}(x)-\alpha\right) \mathbb{1}_{I_{j}^{\phi}}(x) .
$$

Moreover, note that the chain rule implies that for all $x \in E$ we have that

$$
\mathbb{R} \ni u \mapsto\left(\mathcal{N}_{\infty}^{\left(\phi_{1}, \ldots, \phi_{H+j-1}, u, \phi_{H+j+1}, \ldots, \phi_{3 H+1}\right)}(x)-\alpha\right)^{2} \in \mathbb{R}
$$

is differentiable at $\phi_{H+j}$ and

$$
\frac{\partial}{\partial \phi_{H+j}}\left(\mathcal{N}_{\infty}^{\phi}(x)-\alpha\right)^{2}=2 \phi_{2 H+j}\left(\mathcal{N}_{\infty}^{\phi}(x)-\alpha\right) \mathbb{1}_{(0, \infty)}\left(\phi_{j} x+\phi_{H+j}\right)=2 \phi_{2 H+j}\left(\mathcal{N}_{\infty}^{\phi}(x)-\alpha\right) \mathbb{1}_{I_{j}^{\phi}}(x) .
$$

Combining (29), (30), (31), Corollary 2.5, and (7) hence establishes items (i) and (ii). The proof of Lemma 2.7 is thus complete.

Lemma 2.8. Assume Setting 2.1, let $\phi=\left(\phi_{1}, \ldots, \phi_{3 H+1}\right) \in \mathbb{R}^{3 H+1}, j \in\{1,2, \ldots, H\}$, assume $\phi_{j}=\phi_{H+j}=0$, and assume that $\mathcal{L}_{\infty}$ is differentiable at $\phi$. Then $\left(\frac{\partial}{\partial \phi_{j}} \mathcal{L}_{\infty}\right)(\phi)=\mathcal{G}_{j}(\phi)=$ $\left(\frac{\partial}{\partial \phi_{H+j}} \mathcal{L}_{\infty}\right)(\phi)=\mathcal{G}_{H+j}(\phi)=0$.

Proof of Lemma 2.8. Throughout this proof let $\varphi^{h}=\left(\varphi_{1}^{h}, \ldots, \varphi_{3 H+1}^{h}\right) \in \mathbb{R}^{3 H+1}, h=\left(h_{1}, h_{2}\right) \in$ $\mathbb{R}^{2}$, satisfy for all $h=\left(h_{1}, h_{2}\right) \in \mathbb{R}^{2}, k \in\{1,2, \ldots, 3 H+1\} \backslash\{j, H+j\}$ that $\varphi_{j}^{h}=\phi_{j}+h_{1}$, $\varphi_{H+j}^{h}=\phi_{H+j}+h_{2}$, and $\varphi_{k}^{h}=\phi_{k}$. Observe that the assumption that $\mathcal{L}_{\infty}$ is differentiable at $\phi$ ensures that for all $i \in\{j, H+j\}$ it holds that $\mathbb{R} \ni v \mapsto \mathcal{L}_{\infty}\left(\phi_{1}, \ldots, \phi_{i-1}, v, \phi_{i+1}, \ldots, \phi_{3 H+1}\right) \in \mathbb{R}$ is differentiable at $\phi_{i}$. Furthermore, note that for all $h \in(-\infty, 0]^{2}, x \in[0,1]$ it holds that $\mathcal{N}_{\infty}^{\varphi^{h}}(x)=\mathcal{N}_{\infty}^{\phi}(x)$. Hence, we have for all $h \in(-\infty, 0]^{2}$ that $\mathcal{L}_{\infty}\left(\varphi^{h}\right)=\mathcal{L}_{\infty}(\phi)$. This implies that $\left(\frac{\partial}{\partial \phi_{j}} \mathcal{L}_{\infty}\right)(\phi)=\left(\frac{\partial}{\partial \phi_{H+j}} \mathcal{L}_{\infty}\right)(\phi)=0$. Moreover, observe that the assumption that $\phi_{j}=\phi_{H+j}=0$ implies that $I_{j}^{\phi}=\varnothing$. This and $(7)$ demonstrate that $\mathcal{G}_{j}(\phi)=\mathcal{G}_{H+j}(\phi)=0$. Hence, we obtain that $\left(\frac{\partial}{\partial \phi_{j}} \mathcal{L}_{\infty}\right)(\phi)=0=\mathcal{G}_{j}(\phi)$ and $\left(\frac{\partial}{\partial \phi_{H+j}} \mathcal{L}_{\infty}\right)(\phi)=0=\mathcal{G}_{H+j}(\phi)$. This completes the proof of Lemma 2.8 .

Corollary 2.9. Assume Setting 2.1, let $\phi=\left(\phi_{1}, \ldots, \phi_{3 H+1}\right) \in \mathbb{R}^{3 H+1}$, and assume that $\mathcal{L}_{\infty}$ is differentiable at $\phi$. Then $\left(\nabla \mathcal{L}_{\infty}\right)(\phi)=\mathcal{G}(\phi)$.

Proof of Corollary 2.9. Note that the assumption that $\mathcal{L}_{\infty}$ is differentiable at $\phi$ ensures that for all $i \in\{1,2, \ldots, 3 H+1\}$ it holds that $\mathbb{R} \ni v \mapsto \mathcal{L}_{\infty}\left(\phi_{1}, \ldots, \phi_{i-1}, v, \phi_{i+1}, \ldots, \phi_{3 H+1}\right) \in \mathbb{R}$ is differentiable at $\phi_{i}$. Moreover, observe that Lemma 2.6 proves for all $j \in \mathbb{N} \cap(2 H, 3 H+1]$ that $\left(\frac{\partial}{\partial \phi_{j}} \mathcal{L}_{\infty}\right)(\phi)=\mathcal{G}_{j}(\phi)$. In addition, note that Lemma 2.7 shows that for all $j \in\{1,2, \ldots, H\}$ with $\left|\phi_{j}\right|+\left|\phi_{H+j}\right|>0$ it holds that $\left(\frac{\partial}{\partial \phi_{j}} \mathcal{L}_{\infty}\right)(\phi)=\mathcal{G}_{j}(\phi)$ and $\left(\frac{\partial}{\partial \phi_{H+j}} \mathcal{L}_{\infty}\right)(\phi)=\mathcal{G}_{H+j}(\phi)$. On the other hand, observe that Lemma 2.8 ensures that for all $j \in\{1,2, \ldots, H\}$ with $\phi_{j}=\phi_{H+j}=0$ we have that $\left(\frac{\partial}{\partial \phi_{j}} \mathcal{L}_{\infty}\right)(\phi)=0=\mathcal{G}_{j}(\phi)$ and $\left(\frac{\partial}{\partial \phi_{H+j}} \mathcal{L}_{\infty}\right)(\phi)=0=\mathcal{G}_{H+j}(\phi)$. This demonstrates that $\left(\nabla \mathcal{L}_{\infty}\right)(\phi)=\mathcal{G}(\phi)$. The proof of Corollary 2.9 is thus complete.

\subsection{Upper bounds for gradients of the risk functions}

Lemma 2.10. Assume Setting 2.1 and let $\phi \in \mathbb{R}^{3 H+1}$. Then

$$
\|\mathcal{G}(\phi)\|^{2} \leq\left(8\|\phi\|^{2}+4\right) \mathcal{L}_{\infty}(\phi) .
$$


Proof of Lemma 2.10. Throughout this proof let $w_{1}, \ldots, w_{H}, b_{1}, \ldots, b_{H}, v_{1}, \ldots, v_{H}, c \in \mathbb{R}$ satisfy $\phi=\left(w_{1}, \ldots, w_{H}, b_{1}, \ldots, b_{H}, v_{1}, \ldots, v_{H}, c\right)$. Note that Jensen's inequality implies that

$$
\left(\int_{0}^{1}\left|\mathcal{N}_{\infty}^{\phi}(x)-\alpha\right| \mathrm{d} x\right)^{2} \leq \int_{0}^{1}\left(\mathcal{N}_{\infty}^{\phi}(x)-\alpha\right)^{2} \mathrm{~d} x=\mathcal{L}_{\infty}(\phi)
$$

This and (7) ensure that for all $j \in\{1,2, \ldots, H\}$ we have that

$$
\begin{aligned}
\left|\mathcal{G}_{j}(\phi)\right|^{2} & =4\left(v_{j}\right)^{2}\left(\int_{I_{j}^{\phi}} x\left(\mathcal{N}_{\infty}^{\phi}(x)-\alpha\right) \mathrm{d} x\right)^{2} \leq 4\left(v_{j}\right)^{2}\left(\int_{I_{j}^{\phi}}|x|\left|\mathcal{N}_{\infty}^{\phi}(x)-\alpha\right| \mathrm{d} x\right)^{2} \\
& \leq 4\left(v_{j}\right)^{2}\left(\int_{0}^{1}\left|\mathcal{N}_{\infty}^{\phi}(x)-\alpha\right| \mathrm{d} x\right)^{2} \leq 4\left(v_{j}\right)^{2} \mathcal{L}_{\infty}(\phi) .
\end{aligned}
$$

In addition, observe that (7) and (35) assure that for all $j \in\{1,2, \ldots, H\}$ it holds that

$$
\begin{aligned}
\left|\mathcal{G}_{H+j}(\phi)\right|^{2} & =4\left(v_{j}\right)^{2}\left(\int_{I_{j}^{\phi}}\left(\mathcal{N}_{\infty}^{\phi}(x)-\alpha\right) \mathrm{d} x\right)^{2} \\
& \leq 4\left(v_{j}\right)^{2}\left(\int_{0}^{1}\left|\mathcal{N}_{\infty}^{\phi}(x)-\alpha\right| \mathrm{d} x\right)^{2} \leq 4\left(v_{j}\right)^{2} \mathcal{L}_{\infty}(\phi) .
\end{aligned}
$$

Furthermore, note that for all $x \in[0,1], j \in\{1,2, \ldots, H\}$ it holds that $\left|\sigma_{\infty}\left(w_{j} x+b_{j}\right)\right|^{2} \leq$ $\left(\left|w_{j}\right|+\left|b_{j}\right|\right)^{2} \leq 2\left(\left(w_{j}\right)^{2}+\left(b_{j}\right)^{2}\right)$. Combining this and (7) demonstrates for all $j \in\{1,2, \ldots, H\}$ that

$$
\begin{aligned}
\left|\mathcal{G}_{2 H+j}(\phi)\right|^{2} & =4\left(\int_{0}^{1}\left[\sigma_{\infty}\left(w_{j} x+b_{j}\right)\right]\left(\mathcal{N}_{\infty}^{\phi}(x)-\alpha\right) \mathrm{d} x\right)^{2} \\
& \leq 4 \int_{0}^{1}\left|\sigma_{\infty}\left(w_{j} x+b_{j}\right)\right|^{2}\left(\mathcal{N}_{\infty}^{\phi}(x)-\alpha\right)^{2} \mathrm{~d} x \leq 8\left[\left(w_{j}\right)^{2}+\left(b_{j}\right)^{2}\right] \mathcal{L}_{\infty}(\phi) .
\end{aligned}
$$

Finally, observe that (7) and (35) show that

$$
\left|\mathcal{G}_{3 H+1}(\phi)\right|^{2}=4\left(\int_{0}^{1}\left(\mathcal{N}_{\infty}^{\phi}(x)-\alpha\right) \mathrm{d} x\right)^{2} \leq 4 \mathcal{L}_{\infty}(\phi) .
$$

Combining (36)-(39) yields

$$
\begin{aligned}
\|\mathcal{G}(\phi)\|^{2} & \leq\left[\sum_{j=1}^{H}\left(4\left(v_{j}\right)^{2}+4\left(v_{j}\right)^{2}+8\left(w_{j}\right)^{2}+8\left(b_{j}\right)^{2}\right)\right] \mathcal{L}_{\infty}(\phi)+4 \mathcal{L}_{\infty}(\phi) \\
& \leq\left(8\|\phi\|^{2}+4\right) \mathcal{L}_{\infty}(\phi) .
\end{aligned}
$$

The proof of Lemma 2.10 is thus complete.

Corollary 2.11. Assume Setting 2.1 and let $K \subseteq \mathbb{R}^{3 H+1}$ be a compact set. Then $\sup _{\phi \in K}\|\mathcal{G}(\phi)\|<$ $\infty$.

Proof of Corollary 2.11. Note that the fact that $\mathcal{L}_{\infty}$ is continuous ensures that $\sup _{\phi \in K} \mathcal{L}_{\infty}(\phi)<$ $\infty$. Combining this with Lemma 2.10 completes the proof of Corollary 2.11.

\subsection{Properties of Lyapunov type functions}

Proposition 2.12. Assume Setting 2.1 and let $\phi \in \mathbb{R}^{3 H+1}$. Then

$$
\|\phi\|^{2} \leq V(\phi) \leq 3\|\phi\|^{2}+8 \alpha^{2} .
$$


Proof of Proposition 2.12. Observe that $V(\phi)=\|\phi\|^{2}+\left(\mathfrak{c}^{\phi}-2 \alpha\right)^{2} \geq\|\phi\|^{2}$. Furthermore, note that the fact that $\forall x, y \in \mathbb{R}:(x-y)^{2} \leq 2\left(x^{2}+y^{2}\right)$ establishes that

$$
V(\phi) \leq\|\phi\|^{2}+2\left(\mathfrak{c}^{\phi}\right)^{2}+8 \alpha^{2} \leq 3\|\phi\|^{2}+8 \alpha^{2} .
$$

This completes the proof of Proposition 2.12.

Proposition 2.13. Assume Setting 2.1 and let $\phi, \psi \in \mathbb{R}^{3 H+1}$. Then

$$
(\nabla V)(\phi)-(\nabla V)(\psi)=2(\phi-\psi)+\left(0,0, \ldots, 0,2\left(\mathfrak{c}^{\phi}-\mathfrak{c}^{\psi}\right)\right) .
$$

Proof of Proposition 2.13. Observe that for all $\varphi \in \mathbb{R}^{3 H+1}$ it holds that

$$
(\nabla V)(\varphi)=2 \varphi+\left(0,0, \ldots, 0,2\left(\mathfrak{c}^{\varphi}-2 \alpha\right)\right) .
$$

This establishes (43). The proof of Proposition 2.13 is thus complete.

Proposition 2.14. Assume Setting 2.1, let $\mathcal{V}_{1}, \mathcal{V}_{2} \in C\left(\mathbb{R}^{3 H+1}, \mathbb{R}\right)$ satisfy for all $\phi \in \mathbb{R}^{3 H+1}$ that $\mathcal{V}_{1}(\phi)=\left(\mathfrak{c}^{\phi}\right)^{2}-2 \alpha \mathfrak{c}^{\phi}+\sum_{j=1}^{H}\left(\mathfrak{v}_{j}^{\phi}\right)^{2}$ and $\mathcal{V}_{2}(\phi)=\left(\mathfrak{c}^{\phi}\right)^{2}-2 \alpha \mathfrak{c}^{\phi}+\sum_{j=1}^{H}\left[\left(\mathfrak{w}_{j}^{\phi}\right)^{2}+\left(\mathfrak{b}_{j}^{\phi}\right)^{2}\right]$, and let $\phi \in \mathbb{R}^{3 H+1}$. Then

(i) it holds that $\left\langle\left(\nabla \mathcal{V}_{1}\right)(\phi), \mathcal{G}(\phi)\right\rangle=4 \mathcal{L}_{\infty}(\phi)$,

(ii) it holds that $\left\langle\left(\nabla \mathcal{V}_{2}\right)(\phi), \mathcal{G}(\phi)\right\rangle=4 \mathcal{L}_{\infty}(\phi)$, and

(iii) it holds that $\langle(\nabla V)(\phi), \mathcal{G}(\phi)\rangle=8 \mathcal{L}_{\infty}(\phi)$.

Proof of Proposition 2.14. Throughout this proof let $w_{1}, \ldots, w_{H}, b_{1}, \ldots, b_{H}, v_{1}, \ldots, v_{H}, c \in \mathbb{R}$ satisfy $\phi=\left(w_{1}, \ldots, w_{H}, b_{1}, \ldots, b_{H}, v_{1}, \ldots, v_{H}, c\right)$. Note that

$$
\left(\nabla \mathcal{V}_{1}\right)(\phi)=2(\underbrace{0,0, \ldots, 0}_{2 H}, v_{1}, v_{2}, \ldots, v_{H}, c-\alpha) .
$$

This and (7) imply that

$$
\begin{aligned}
& \left\langle\left(\nabla \mathcal{V}_{1}\right)(\phi), \mathcal{G}(\phi)\right\rangle \\
& =4\left[\sum_{j=1}^{H} v_{j} \int_{0}^{1}\left[\sigma_{\infty}\left(w_{j} x+b_{j}\right)\right]\left(\mathcal{N}_{\infty}^{\phi}(x)-\alpha\right) \mathrm{d} x\right]+4(c-\alpha) \int_{0}^{1}\left(\mathcal{N}_{\infty}^{\phi}(x)-\alpha\right) \mathrm{d} x \\
& =4 \int_{0}^{1}\left(\left[\sum_{j=1}^{H} v_{j} \sigma_{\infty}\left(w_{j} x+b_{j}\right)\right]+c-\alpha\right)\left(\mathcal{N}_{\infty}^{\phi}(x)-\alpha\right) \mathrm{d} x \\
& =4 \int_{0}^{1}\left(\mathcal{N}_{\infty}^{\phi}(x)-\alpha\right)^{2} \mathrm{~d} x=4 \mathcal{L}_{\infty}(\phi) .
\end{aligned}
$$

This proves item (i). Next observe that

$$
\left(\nabla \mathcal{V}_{2}\right)(\phi)=2(w_{1}, w_{2}, \ldots, w_{H}, b_{1}, b_{2}, \ldots, b_{H}, \underbrace{0,0, \ldots, 0}_{H}, c-\alpha)
$$

Combining this and (7) demonstrates that

$$
\begin{aligned}
& \left\langle\left(\nabla \mathcal{V}_{2}\right)(\phi), \mathcal{G}(\phi)\right\rangle \\
& =4\left[\sum_{j=1}^{H} v_{j} \int_{I_{j}^{\phi}}\left(w_{j} x+b_{j}\right)\left(\mathcal{N}_{\infty}^{\phi}(x)-\alpha\right) \mathrm{d} x\right]+4(c-\alpha) \int_{0}^{1}\left(\mathcal{N}_{\infty}^{\phi}(x)-\alpha\right) \mathrm{d} x \\
& =4\left[\sum_{j=1}^{H} v_{j} \int_{0}^{1}\left[\sigma_{\infty}\left(w_{j} x+b_{j}\right)\right]\left(\mathcal{N}_{\infty}^{\phi}(x)-\alpha\right) \mathrm{d} x\right]+4(c-\alpha) \int_{0}^{1}\left(\mathcal{N}_{\infty}^{\phi}(x)-\alpha\right) \mathrm{d} x \\
& =4 \int_{0}^{1}\left(\left[\sum_{j=1}^{H} v_{j} \sigma_{\infty}\left(w_{j} x+b_{j}\right)\right]+c-\alpha\right)\left(\mathcal{N}_{\infty}^{\phi}(x)-\alpha\right) \mathrm{d} x \\
& =4 \int_{0}^{1}\left(\mathcal{N}_{\infty}^{\phi}(x)-\alpha\right)^{2} \mathrm{~d} x=4 \mathcal{L}_{\infty}(\phi) .
\end{aligned}
$$


This establishes item (ii). Furthermore, note that $\mathcal{V}_{1}(\phi)+\mathcal{V}_{2}(\phi)=V(\phi)-4 \alpha^{2}$. This ensures that $\left(\nabla \mathcal{V}_{1}\right)(\phi)+\left(\nabla \mathcal{V}_{2}\right)(\phi)=(\nabla V)(\phi)$, which proves item (iii). The proof of Proposition 2.14 is thus complete.

Corollary 2.15. Assume Setting 2.1 and let $\phi \in \mathbb{R}^{3 H+1}$. Then it holds that $\|\mathcal{G}(\phi)\|=0$ if and only if $\mathcal{L}_{\infty}(\phi)=0$.

Proof of Corollary 2.15. Assume first that $\|\mathcal{G}(\phi)\|=0$. Then Proposition 2.14 implies that $8 \mathcal{L}_{\infty}(\phi)=\langle(\nabla V)(\phi), \mathcal{G}(\phi)\rangle=0$. Next assume $\mathcal{L}_{\infty}(\phi)=\int_{0}^{1}\left(\mathscr{N}_{\infty}^{\phi}(x)-\alpha\right)^{2} \mathrm{~d} x=0$. The fact that $\mathcal{N}_{\infty}^{\phi} \in C(\mathbb{R}, \mathbb{R})$ then implies that it holds for all $x \in[0,1]$ that $\mathcal{N}_{\infty}^{\phi}(x)=\alpha$. Hence, (7) demonstrates that $\mathcal{G}(\phi)=0 \in \mathbb{R}^{3 H+1}$ and therefore $\|\mathcal{G}(\phi)\|=0$. This completes the proof of Corollary 2.15.

\section{Convergence analysis for gradient flow processes}

In this section we employ the findings from Section 2 to establish in Theorem 3.7 below that the risks of the considered time-continuous gradient flow processes converge to zero. Our proof of Theorem 3.7 uses the deterministic Itô type formula for the Lyapunov function $V: \mathbb{R}^{3 H+1} \rightarrow \mathbb{R}$ from Setting 2.1, which we establish in Lemma 3.2 in Subsection 3.1 below, as well as the deterministic Itô type formula for the risk function $\mathcal{L}_{\infty}: \mathbb{R}^{3 H+1} \rightarrow \mathbb{R}$ from Setting 2.1, which we establish in Lemma 3.5 in Subsection 3.2 below.

Our proof of the deterministic Itô type formula for the Lyapunov function $V: \mathbb{R}^{3 H+1} \rightarrow \mathbb{R}$ in Lemma 3.2, in turn, is based on the fact that the function $V: \mathbb{R}^{3 H+1} \rightarrow \mathbb{R}$ from Setting 2.1 satisfies the Lyapunov property in item (iii) in Proposition 2.14 as well as on the well-known deterministic Itô-type formula for continuously differentiable functions in Lemma 3.1 in Subsection 3.1 below. We include in this section a detailed proof for Lemma 3.1 only for completeness.

In contrast to Lemma 3.2, the deterministic Itô type formula for the risk function $\mathcal{L}_{\infty}: \mathbb{R}^{3 H+1}$ $\rightarrow \mathbb{R}$ in Lemma 3.5 can not be proved through an application of Lemma 3.1 as the risk function $\mathcal{L}_{\infty}: \mathbb{R}^{3 H+1} \rightarrow \mathbb{R}$ fails to be differentiable. Instead we prove Lemma 3.5 through an approximation argument by employing the mollified rectifier functions $\sigma_{r} \in C^{\infty}(\mathbb{R}, \mathbb{R}), r \in[1, \infty)$, and their corresponding risk functions $\mathcal{L}_{r}: \mathbb{R}^{3 H+1} \rightarrow \mathbb{R}, r \in[1, \infty)$, from Setting 2.1.

\subsection{Deterministic Itô formulas for Lyapunov type functions}

Lemma 3.1. Let $T \in(0, \infty), n \in \mathbb{N}, \Theta \in C\left([0, T], \mathbb{R}^{n}\right), F \in C^{1}\left(\mathbb{R}^{n}, \mathbb{R}\right)$, let $\vartheta:[0, T] \rightarrow \mathbb{R}^{n}$ be a bounded measurable function, and assume for all $t \in[0, T]$ that

$$
\Theta_{t}=\Theta_{0}+\int_{0}^{t} \vartheta_{s} \mathrm{~d} s
$$

Then it holds for all $t \in[0, T]$ that

$$
F\left(\Theta_{t}\right)=F\left(\Theta_{0}\right)+\int_{0}^{t}\left(F^{\prime}\left(\Theta_{s}\right)\right) \vartheta_{s} \mathrm{~d} s .
$$

Proof of Lemma 3.1. Observe that the fact that $\vartheta$ is bounded proves that $\Theta$ is Lipschitz continuous. Combining this and Rademacher's theorem shows that there exists a measurable set $E \subseteq[0, T]$ which satisfies that $\int_{[0, T] \backslash E} 1 \mathrm{~d} s=0$, which satisfies for all $t \in E$ that $[0, T] \ni s \mapsto$ $\Theta_{s} \in \mathbb{R}^{n}$ is differentiable at $t$, and which satisfies for all $t \in E$ that $\frac{\mathrm{d}}{\mathrm{d} t} \Theta_{t}=\vartheta_{t}$. This and the chain rule demonstrate that for all $t \in E$ it holds that $[0, T] \ni s \mapsto F\left(\Theta_{s}\right) \in \mathbb{R}$ is differentiable at $t$ and that $\frac{\mathrm{d}}{\mathrm{d} t}\left(F\left(\Theta_{t}\right)\right)=\left(F^{\prime}\left(\Theta_{t}\right)\right) \vartheta_{t}$. Furthermore, note that the fact that $\Theta$ is Lipschitz continuous and the fact that $F$ is continuously differentiable establish that $[0, T] \ni t \mapsto F\left(\Theta_{t}\right) \in \mathbb{R}$ 
is Lipschitz continuous. Hence, we obtain that $[0, T] \ni t \mapsto F\left(\Theta_{t}\right) \in \mathbb{R}$ is absolutely continuous. This shows for all $t \in[0, T]$ that

$$
F\left(\Theta_{t}\right)=F\left(\Theta_{0}\right)+\int_{0}^{t}\left(F^{\prime}\left(\Theta_{s}\right)\right) \vartheta_{s} \mathrm{~d} s .
$$

The proof of Lemma 3.1 is thus complete.

Lemma 3.2. Assume Setting 2.1, let $T \in(0, \infty)$, and let $\Theta \in C\left([0, T], \mathbb{R}^{3 H+1}\right)$ satisfy for all $t \in[0, T]$ that $\Theta_{t}=\Theta_{0}-\int_{0}^{t} \mathcal{G}\left(\Theta_{s}\right) \mathrm{d} s$. Then it holds for all $t \in[0, T]$ that $V\left(\Theta_{t}\right)=$ $V\left(\Theta_{0}\right)-8 \int_{0}^{t} \mathcal{L}_{\infty}\left(\Theta_{s}\right) \mathrm{d} s$.

Proof of Lemma 3.2. Observe that Corollary 2.11 and the assumption that $\Theta \in C\left([0, T], \mathbb{R}^{3 H+1}\right)$ imply that $[0, T] \ni t \mapsto \mathcal{G}\left(\Theta_{t}\right) \in \mathbb{R}^{3 H+1}$ is bounded. Combining this, the fact that $V \in$ $C^{\infty}\left(\mathbb{R}^{3 H+1}, \mathbb{R}\right)$, Proposition 2.3, Lemma 3.1, and Proposition 2.14 demonstrates that for all $t \in[0, T]$ we have that

$$
V\left(\Theta_{t}\right)-V\left(\Theta_{0}\right)=-\int_{0}^{t}\left\langle(\nabla V)\left(\Theta_{s}\right), \mathcal{G}\left(\Theta_{s}\right)\right\rangle \mathrm{d} s=-8 \int_{0}^{t} \mathcal{L}_{\infty}\left(\Theta_{s}\right) \mathrm{d} s .
$$

The proof of Lemma 3.2 is thus complete.

Corollary 3.3. Assume Setting 2.1 and let $\Theta \in C\left([0, \infty), \mathbb{R}^{3 H+1}\right)$ satisfy for all $t \in[0, \infty)$ that $\Theta_{t}=\Theta_{0}-\int_{0}^{t} \mathcal{G}\left(\Theta_{s}\right) \mathrm{d} s$. Then $\sup _{t \in[0, \infty)}\left\|\Theta_{t}\right\| \leq\left[V\left(\Theta_{0}\right)\right]^{1 / 2}<\infty$.

Proof of Corollary 3.3. Note that Proposition 2.12 implies for all $t \in[0, \infty)$ that $\left\|\Theta_{t}\right\| \leq$ $\left[V\left(\Theta_{t}\right)\right]^{1 / 2}$. Furthermore, observe that Lemma 3.2 and the fact that $\forall \phi \in \mathbb{R}^{3 H+1}: \mathcal{L}_{\infty}(\phi) \geq 0$ demonstrate for all $t \in[0, \infty)$ that $V\left(\Theta_{t}\right) \leq V\left(\Theta_{0}\right)$. This completes the proof of Corollary 3.3.

\subsection{Deterministic Itô formulas for risk functions}

Lemma 3.4. Assume Setting 2.1 and let $K \subseteq \mathbb{R}^{3 H+1}$ be a compact set. Then $\sup _{\phi \in K} \sup _{r \in[1, \infty)}$ $\left\|\left(\nabla \mathcal{L}_{r}\right)(\phi)\right\|<\infty$.

Proof of Lemma 3.4. Note that Proposition 2.2 demonstrates for all $r \in[1, \infty), \phi=\left(w_{1}, \ldots\right.$, $\left.w_{H}, b_{1}, \ldots, b_{H}, v_{1}, \ldots, v_{H}, c\right) \in \mathbb{R}^{3 H+1}, x \in[0,1]$ that

$$
\left|\mathcal{N}_{r}^{\phi}(x)\right| \leq|c|+\sum_{j=1}^{H}\left|v_{j}\right|\left(\sigma_{\infty}\left(w_{j} x+b_{j}\right)+1\right) \leq|c|+\sum_{j=1}^{H}\left|v_{j}\right|\left(\left|w_{j}\right|+\left|b_{j}\right|+1\right) .
$$

Hence, we obtain for all $r \in[1, \infty), \phi=\left(w_{1}, \ldots, w_{H}, b_{1}, \ldots, b_{H}, v_{1}, \ldots, v_{H}, c\right) \in \mathbb{R}^{3 H+1}$ that

$$
\mathcal{L}_{r}(\phi) \leq \int_{0}^{1}\left(|\alpha|+\left|\mathcal{N}_{r}^{\phi}(x)\right|\right)^{2} \mathrm{~d} x \leq\left(|\alpha|+|c|+\sum_{j=1}^{H}\left|v_{j}\right|\left(\left|w_{j}\right|+\left|b_{j}\right|+1\right)\right)^{2} .
$$

This implies that $\sup _{\phi \in K} \sup _{r \in[1, \infty)} \mathcal{L}_{r}(\phi)<\infty$. Next observe that (6) and the Cauchy-Schwarz inequality demonstrate that for all $r \in[1, \infty), \phi=\left(w_{1}, \ldots, w_{H}, b_{1}, \ldots, b_{H}, v_{1}, \ldots, v_{H}, c\right) \in$ $\mathbb{R}^{3 H+1}$ it holds that

$$
\left|\left(\frac{\partial}{\partial c} \mathcal{L}_{r}\right)(\phi)\right| \leq 2 \int_{0}^{1}\left|\mathcal{N}_{r}^{\phi}(x)-\alpha\right| \mathrm{d} x \leq 2 \sqrt{\mathcal{L}_{r}(\phi)} .
$$

Furthermore, note that the Cauchy-Schwarz inequality, Proposition 2.2, and (6) prove that for all $r \in[1, \infty), \phi=\left(w_{1}, \ldots, w_{H}, b_{1}, \ldots, b_{H}, v_{1}, \ldots, v_{H}, c\right) \in \mathbb{R}^{3 H+1}, j \in\{1,2, \ldots, H\}$ it holds that

$$
\begin{aligned}
\left|\left(\frac{\partial}{\partial w_{j}} \mathcal{L}_{r}\right)(\phi)\right| & \leq 2\left|v_{j}\right| \int_{0}^{1}\left|x\left(\sigma_{r}\right)^{\prime}\left(w_{j} x+b_{j}\right)\right|\left|\mathcal{N}_{r}^{\phi}(x)-\alpha\right| \mathrm{d} x \\
& \leq 2\left|v_{j}\right| \int_{0}^{1}\left|\mathcal{N}_{r}^{\phi}(x)-\alpha\right| \mathrm{d} x \leq 2\left|v_{j}\right| \sqrt{\mathcal{L}_{r}(\phi)}
\end{aligned}
$$


and

$$
\begin{aligned}
\left|\left(\frac{\partial}{\partial b_{j}} \mathcal{L}_{r}\right)(\phi)\right| & \leq 2\left|v_{j}\right| \int_{0}^{1}\left|\left(\sigma_{r}\right)^{\prime}\left(w_{j} x+b_{j}\right)\right|\left|\mathcal{N}_{r}^{\phi}(x)-\alpha\right| \mathrm{d} x \\
& \leq 2\left|v_{j}\right| \int_{0}^{1}\left|\mathcal{N}_{r}^{\phi}(x)-\alpha\right| \mathrm{d} x \leq 2\left|v_{j}\right| \sqrt{\mathcal{L}_{r}(\phi)} .
\end{aligned}
$$

In addition, observe that the Cauchy-Schwarz inequality, Proposition 2.2, and (6) demonstrate that for all $r \in[1, \infty), \phi=\left(w_{1}, \ldots, w_{H}, b_{1}, \ldots, b_{H}, v_{1}, \ldots, v_{H}, c\right) \in \mathbb{R}^{3 H+1}, j \in\{1,2, \ldots, H\}$ it holds that

$$
\begin{aligned}
\left|\left(\frac{\partial}{\partial v_{j}} \mathcal{L}_{r}\right)(\phi)\right| & \leq 2 \int_{0}^{1}\left[\sigma_{r}\left(w_{j} x+b_{j}\right)\right]\left|\mathcal{N}_{r}^{\phi}(x)-\alpha\right| \mathrm{d} x \\
& \leq 2\left(1+\left|w_{j}\right|+\left|b_{j}\right|\right) \int_{0}^{1}\left|\mathcal{N}_{r}^{\phi}(x)-\alpha\right| \mathrm{d} x \\
& \leq 2\left(1+\left|w_{j}\right|+\left|b_{j}\right|\right) \sqrt{\mathcal{L}_{r}(\phi)} .
\end{aligned}
$$

This, (55), (56), and (57) show that for all $r \in[1, \infty), \phi=\left(w_{1}, \ldots, w_{H}, b_{1}, \ldots, b_{H}, v_{1}, \ldots\right.$, $\left.v_{H}, c\right) \in \mathbb{R}^{3 H+1}$ it holds that

$$
\left\|\left(\nabla \mathcal{L}_{r}\right)(\phi)\right\|^{2} \leq\left[4+\sum_{j=1}^{H}\left(8\left(v_{j}\right)^{2}+4\left(1+\left|w_{j}\right|+\left|b_{j}\right|\right)^{2}\right)\right] \mathcal{L}_{r}(\phi) .
$$

Combining this with the fact that $\sup _{\phi \in K} \sup _{r \in[1, \infty)} \mathcal{L}_{r}(\phi)<\infty$ establishes that

$$
\sup _{\phi \in K} \sup _{r \in[1, \infty)}\left\|\left(\nabla \mathcal{L}_{r}\right)(\phi)\right\|^{2}<\infty .
$$

The proof of Lemma 3.4 is thus complete.

Lemma 3.5. Assume Setting 2.1, let $T \in(0, \infty)$, and let $\Theta \in C\left([0, T], \mathbb{R}^{3 H+1}\right)$ satisfy for all $t \in[0, T]$ that $\Theta_{t}=\Theta_{0}-\int_{0}^{t} \mathcal{G}\left(\Theta_{s}\right) \mathrm{d} s$. Then it holds for all $t \in[0, T]$ that $\mathcal{L}_{\infty}\left(\Theta_{t}\right)=$ $\mathcal{L}_{\infty}\left(\Theta_{0}\right)-\int_{0}^{t}\left\|\mathcal{G}\left(\Theta_{s}\right)\right\|^{2} \mathrm{~d} s$.

Proof of Lemma 3.5. Note that Lemma 3.1 and item (i) in Proposition 2.3 demonstrate that for all $r \in[1, \infty), t \in[0, T]$ it holds that

$$
\mathcal{L}_{r}\left(\Theta_{t}\right)-\mathcal{L}_{r}\left(\Theta_{0}\right)=-\int_{0}^{t}\left\langle\left(\nabla \mathcal{L}_{r}\right)\left(\Theta_{s}\right), \mathcal{G}\left(\Theta_{s}\right)\right\rangle \mathrm{d} s
$$

Next observe that Proposition 2.3 proves that for all $t \in[0, T]$ it holds that $\lim _{r \rightarrow \infty}\left(\mathcal{L}_{r}\left(\Theta_{t}\right)-\right.$ $\left.\mathcal{L}_{r}\left(\Theta_{0}\right)\right)=\mathcal{L}_{\infty}\left(\Theta_{t}\right)-\mathcal{L}\left(\Theta_{0}\right)$. Furthermore, note that Proposition 2.3 ensures that for all $s \in$ $[0, T]$ we have that $\lim _{r \rightarrow \infty}\left\langle\left(\nabla \mathcal{L}_{r}\right)\left(\Theta_{s}\right), \mathcal{G}\left(\Theta_{s}\right)\right\rangle=\left\langle\mathcal{G}\left(\Theta_{s}\right), \mathcal{G}\left(\Theta_{s}\right)\right\rangle=\left\|\mathcal{G}\left(\Theta_{s}\right)\right\|^{2}$. In addition, observe that the assumption that $\Theta \in C\left([0, T], \mathbb{R}^{3 H+1}\right)$ implies that there exists a compact set $K \subseteq \mathbb{R}^{3 H+1}$ such that $\forall s \in[0, T]: \Theta_{s} \in K$. Combining this, the Cauchy-Schwarz inequality, Corollary 2.11, and Lemma 3.4 shows that

$$
\begin{aligned}
& \sup _{r \in[1, \infty)} \sup _{s \in[0, T]}\left|\left\langle\left(\nabla \mathcal{L}_{r}\right)\left(\Theta_{s}\right), \mathcal{G}\left(\Theta_{s}\right)\right\rangle\right| \\
& \leq \sup _{r \in[1, \infty)} \sup _{\phi \in K}\left|\left\langle\left(\nabla \mathcal{L}_{r}\right)(\phi), \mathcal{G}(\phi)\right\rangle\right| \\
& \leq \sup _{r \in[1, \infty)} \sup _{\phi \in K}\left(\left\|\left(\nabla \mathcal{L}_{r}\right)(\phi)\right\|\|\mathcal{G}(\phi)\|\right)<\infty
\end{aligned}
$$

The dominated convergence theorem hence proves that for all $t \in[0, T]$ we have that

$$
\lim _{r \rightarrow \infty}\left[\int_{0}^{t}\left\langle\left(\nabla \mathcal{L}_{r}\right)\left(\Theta_{s}\right), \mathcal{G}\left(\Theta_{s}\right)\right\rangle \mathrm{d} s\right]=\int_{0}^{t}\left[\lim _{r \rightarrow \infty}\left\langle\left(\nabla \mathcal{L}_{r}\right)\left(\Theta_{s}\right), \mathcal{G}\left(\Theta_{s}\right)\right\rangle\right] \mathrm{d} s=\int_{0}^{t}\left\|\mathcal{G}\left(\Theta_{s}\right)\right\|^{2} \mathrm{~d} s .
$$

Combining this with (61) completes the proof of Lemma 3.5. 


\subsection{Convergence of the risks of gradient flow processes}

Lemma 3.6. Assume Setting 2.1 and let $\Theta \in C\left([0, \infty), \mathbb{R}^{3 H+1}\right)$ satisfy for all $t \in[0, \infty)$ that $\Theta_{t}=\Theta_{0}-\int_{0}^{t} \mathcal{G}\left(\Theta_{s}\right) \mathrm{d} s$. Then it holds that $[0, \infty) \ni t \mapsto \mathcal{L}_{\infty}\left(\Theta_{t}\right) \in[0, \infty)$ is non-increasing.

Proof of Lemma 3.6. This is an immediate consequence of Lemma 3.5.

Theorem 3.7. Assume Setting 2.1 and let $\Theta \in C\left([0, \infty), \mathbb{R}^{3 H+1}\right)$ satisfy for all $t \in[0, \infty)$ that $\Theta_{t}=\Theta_{0}-\int_{0}^{t} \mathcal{G}\left(\Theta_{s}\right) \mathrm{d} s$. Then

(i) it holds that $\sup _{t \in[0, \infty)}\left\|\Theta_{t}\right\| \leq\left[V\left(\Theta_{0}\right)\right]^{1 / 2}<\infty$,

(ii) it holds for all $t \in(0, \infty)$ that $\mathcal{L}_{\infty}\left(\Theta_{t}\right) \leq \frac{V\left(\Theta_{0}\right)}{8 t}$, and

(iii) it holds that $\lim \sup _{t \rightarrow \infty} \mathcal{L}_{\infty}\left(\Theta_{t}\right)=0$.

Proof of Theorem 3.7. Note that Corollary 3.3 establishes item (i). Next observe that Lemma 3.2 and Lemma 3.6 prove that for all $t \in[0, \infty)$ it holds that

$$
t \mathcal{L}_{\infty}\left(\Theta_{t}\right)=\int_{0}^{t} \mathcal{L}_{\infty}\left(\Theta_{t}\right) \mathrm{d} s \leq \int_{0}^{t} \mathcal{L}_{\infty}\left(\Theta_{s}\right) \mathrm{d} s=\frac{V\left(\Theta_{0}\right)-V\left(\Theta_{t}\right)}{8} \leq \frac{V\left(\Theta_{0}\right)}{8}<\infty
$$

Hence, we obtain for all $t \in(0, \infty)$ that

$$
\mathcal{L}_{\infty}\left(\Theta_{t}\right) \leq \frac{V\left(\Theta_{0}\right)}{8 t}
$$

This establishes items (ii) and (iii). The proof of Theorem 3.7 is thus complete.

\section{Convergence analysis for gradient descent processes}

In this section we use the findings from Section 2 to prove in Theorem 4.4 in Subsection 4.2 below that the risks of the considered time-discrete GD processes converge to zero. Our proof of Theorem 4.4 uses the fact that the function $V: \mathbb{R}^{3 H+1} \rightarrow \mathbb{R}$ from Setting 2.1 is also a Lyapunov function for the considered time-discrete GD processes, which we establish in Lemma 3.6 below. Moreover, in Subsection 4.3 below we apply Theorem 4.4 to establish in Corollary 4.6 that also the expectations of risks of the time-discrete GD processes with random initializations do converge to zero.

\subsection{Lyapunov type estimates for gradient descent processes}

Lemma 4.1. Assume Setting 2.1, let $\gamma \in(0, \infty)$, and let $\Theta=\left(\Theta_{n}\right)_{n \in \mathbb{N}_{0}}=\left(\left(\Theta_{n}^{1}, \ldots, \Theta_{n}^{3 H+1}\right)\right)_{n \in \mathbb{N}_{0}}$ : $\mathbb{N}_{0} \rightarrow \mathbb{R}^{3 H+1}$ satisfy for all $n \in \mathbb{N}_{0}$ that $\Theta_{n+1}=\Theta_{n}-\gamma \mathcal{G}\left(\Theta_{n}\right)$. Then it holds for all $n \in \mathbb{N}_{0}$ that

$$
V\left(\Theta_{n+1}\right)-V\left(\Theta_{n}\right) \leq-8 \gamma \mathcal{L}_{\infty}\left(\Theta_{n}\right)+2 \gamma^{2}\left\|\mathcal{G}\left(\Theta_{n}\right)\right\|^{2} .
$$

Proof of Lemma 4.1. Throughout this proof let $n \in \mathbb{N}_{0}$ be arbitrary and let $g: \mathbb{R} \rightarrow \mathbb{R}$ satisfy for all $t \in \mathbb{R}$ that $g(t)=V\left(t \Theta_{n+1}+(1-t) \Theta_{n}\right)$. The fact that $V$ is continuously differentiable establishes that $g$ is continuously differentiable. The fundamental theorem of calculus and the 
chain rule hence ensure that

$$
\begin{aligned}
V\left(\Theta_{n+1}\right)-V\left(\Theta_{n}\right)=g(1)-g(0)=\int_{0}^{1} g^{\prime}(t) \mathrm{d} t \\
=\int_{0}^{1}\left\langle(\nabla V)\left(t \Theta_{n+1}+(1-t) \Theta_{n}\right), \Theta_{n+1}-\Theta_{n}\right\rangle \mathrm{d} t \\
=-\gamma \int_{0}^{1}\left\langle(\nabla V)\left(t \Theta_{n+1}+(1-t) \Theta_{n}\right), \mathcal{G}\left(\Theta_{n}\right)\right\rangle \mathrm{d} t \\
=-\gamma \int_{0}^{1}\left\langle(\nabla V)\left(\Theta_{n}\right), \mathcal{G}\left(\Theta_{n}\right)\right\rangle \mathrm{d} t \\
\quad-\gamma \int_{0}^{1}\left\langle(\nabla V)\left(t \Theta_{n+1}+(1-t) \Theta_{n}\right)-(\nabla V)\left(\Theta_{n}\right), \mathcal{G}\left(\Theta_{n}\right)\right\rangle \mathrm{d} t .
\end{aligned}
$$

Next note that Proposition 2.14 implies that $\left\langle(\nabla V)\left(\Theta_{n}\right), \mathcal{G}\left(\Theta_{n}\right)\right\rangle=8 \mathcal{L}_{\infty}\left(\Theta_{n}\right)$. Furthermore, observe that Proposition 2.13 establishes for all $t \in[0,1]$ that

$$
\begin{aligned}
& \left\langle(\nabla V)\left(t \Theta_{n+1}+(1-t) \Theta_{n}\right)-(\nabla V)\left(\Theta_{n}\right), \mathcal{G}\left(\Theta_{n}\right)\right\rangle \\
& =\left\langle(\nabla V)\left(t\left(\Theta_{n+1}-\Theta_{n}\right)+\Theta_{n}\right)-(\nabla V)\left(\Theta_{n}\right), \mathcal{G}\left(\Theta_{n}\right)\right\rangle \\
& =2 t\left\langle\Theta_{n+1}-\Theta_{n}, \mathcal{G}\left(\Theta_{n}\right)\right\rangle+2 t\left(\Theta_{n+1}^{3 H+1}-\Theta_{n}^{3 H+1}\right) \mathcal{G}_{3 H+1}\left(\Theta_{n}\right) \\
& =-2 t \gamma\left\|\mathcal{G}\left(\Theta_{n}\right)\right\|^{2}-2 t \gamma\left|\mathcal{G}_{3 H+1}\left(\Theta_{n}\right)\right|^{2} \geq-4 t \gamma\left\|\mathcal{G}\left(\Theta_{n}\right)\right\|^{2} .
\end{aligned}
$$

Hence, we obtain that

$$
\begin{aligned}
V\left(\Theta_{n+1}\right)-V\left(\Theta_{n}\right) & \leq-8 \gamma \mathcal{L}_{\infty}\left(\Theta_{n}\right)+4 \gamma^{2} \int_{0}^{1} t\left\|\mathcal{G}\left(\Theta_{n}\right)\right\|^{2} \mathrm{~d} t \\
& =-8 \gamma \mathcal{L}_{\infty}\left(\Theta_{n}\right)+2 \gamma^{2}\left\|\mathcal{G}\left(\Theta_{n}\right)\right\|^{2} .
\end{aligned}
$$

The proof of Lemma 4.1 is thus complete.

Corollary 4.2. Assume Setting 2.1, let $\gamma \in(0, \infty)$, and let $\Theta=\left(\Theta_{n}\right)_{n \in \mathbb{N}_{0}}: \mathbb{N}_{0} \rightarrow \mathbb{R}^{3 H+1}$ satisfy for all $n \in \mathbb{N}_{0}$ that $\Theta_{n+1}=\Theta_{n}-\gamma \mathcal{G}\left(\Theta_{n}\right)$. Then it holds for all $n \in \mathbb{N}_{0}$ that

$$
V\left(\Theta_{n+1}\right)-V\left(\Theta_{n}\right) \leq 8\left(-\gamma+\gamma^{2}\left(2 V\left(\Theta_{n}\right)+1\right)\right) \mathcal{L}_{\infty}\left(\Theta_{n}\right) .
$$

Proof of Corollary 4.2. Note that Lemma 2.10 and Proposition 2.12 imply for all $n \in \mathbb{N}_{0}$ that

$$
\begin{aligned}
\left\|\mathcal{G}\left(\Theta_{n}\right)\right\|^{2} & \leq\left(8\left\|\Theta_{n}\right\|^{2}+4\right) \mathcal{L}_{\infty}\left(\Theta_{n}\right)=4\left(2\left\|\Theta_{n}\right\|^{2}+1\right) \mathcal{L}_{\infty}\left(\Theta_{n}\right) \\
& \leq 4\left(2 V\left(\Theta_{n}\right)+1\right) \mathcal{L}_{\infty}\left(\Theta_{n}\right) .
\end{aligned}
$$

Combining this and Lemma 4.1 ensures that for all $n \in \mathbb{N}_{0}$ we have that

$$
\begin{aligned}
V\left(\Theta_{n+1}\right)-V\left(\Theta_{n}\right) & \leq-8 \gamma \mathcal{L}_{\infty}\left(\Theta_{n}\right)+8 \gamma^{2}\left(2 V\left(\Theta_{n}\right)+1\right) \mathcal{L}_{\infty}\left(\Theta_{n}\right) \\
& =8\left(-\gamma+\gamma^{2}\left(2 V\left(\Theta_{n}\right)+1\right)\right) \mathcal{L}_{\infty}\left(\Theta_{n}\right) .
\end{aligned}
$$

The proof of Corollary 4.2 is thus complete.

Lemma 4.3. Assume Setting 2.1, let $\gamma \in(0, \infty)$, and let $\Theta=\left(\Theta_{n}\right)_{n \in \mathbb{N}_{0}}: \mathbb{N}_{0} \rightarrow \mathbb{R}^{3 H+1}$ satisfy for all $n \in \mathbb{N}_{0}$ that $\Theta_{n+1}=\Theta_{n}-\gamma \mathcal{G}\left(\Theta_{n}\right)$ and $\gamma \leq\left(4 V\left(\Theta_{0}\right)+2\right)^{-1}$. Then it holds for all $n \in \mathbb{N}_{0}$ that $V\left(\Theta_{n+1}\right)-V\left(\Theta_{n}\right) \leq-4 \gamma \mathcal{L}_{\infty}\left(\Theta_{n}\right) \leq 0$.

Proof of Lemma 4.3. We prove the statement by induction on $n \in \mathbb{N}_{0}$. Observe that Corollary 4.2 implies that

$$
\begin{aligned}
V\left(\Theta_{1}\right)-V\left(\Theta_{0}\right) & \leq\left(-8 \gamma+8 \gamma^{2}\left(2 V\left(\Theta_{0}\right)+1\right)\right) \mathcal{L}_{\infty}\left(\Theta_{0}\right) \\
& \leq\left(-8 \gamma+8 \gamma\left[\frac{2 V\left(\Theta_{0}\right)+1}{4 V\left(\Theta_{0}\right)+2}\right]\right) \mathcal{L}_{\infty}\left(\Theta_{0}\right)=-4 \gamma \mathcal{L}_{\infty}\left(\Theta_{0}\right) \leq 0
\end{aligned}
$$


This establishes the assertion in the base case $n=0$. For the induction step let $n \in \mathbb{N}$ satisfy for all $m \in\{0,1, \ldots, n-1\}$ that

$$
V\left(\Theta_{m+1}\right)-V\left(\Theta_{m}\right) \leq-4 \gamma \mathcal{L}_{\infty}\left(\Theta_{m}\right) \leq 0 .
$$

Note that (74) shows that $V\left(\Theta_{n}\right) \leq V\left(\Theta_{n-1}\right) \leq \cdots \leq V\left(\Theta_{0}\right)$. The assumption that $\gamma \leq$ $\left(4 V\left(\Theta_{0}\right)+2\right)^{-1}$ hence ensures that $\gamma \leq\left(4 V\left(\Theta_{0}\right)+2\right)^{-1} \leq\left(4 V\left(\Theta_{n}\right)+2\right)^{-1}$. Combining this and Corollary 4.2 demonstrates that

$$
\begin{aligned}
V\left(\Theta_{n+1}\right)-V\left(\Theta_{n}\right) & \leq\left(-8 \gamma+8 \gamma^{2}\left(2 V\left(\Theta_{n}\right)+1\right)\right) \mathcal{L}_{\infty}\left(\Theta_{n}\right) \\
& \leq\left(-8 \gamma+8 \gamma\left[\frac{2 V\left(\Theta_{n}\right)+1}{4 V\left(\Theta_{n}\right)+2}\right]\right) \mathcal{L}_{\infty}\left(\Theta_{n}\right)=-4 \gamma \mathcal{L}_{\infty}\left(\Theta_{n}\right) \leq 0
\end{aligned}
$$

This completes the proof of Lemma 4.3.

\subsection{Convergence of the risks of gradient descent processes}

Theorem 4.4. Assume Setting 2.1, let $\gamma \in(0, \infty)$, and let $\Theta=\left(\Theta_{n}\right)_{n \in \mathbb{N}_{0}}: \mathbb{N}_{0} \rightarrow \mathbb{R}^{3 H+1}$ satisfy for all $n \in \mathbb{N}_{0}$ that $\Theta_{n+1}=\Theta_{n}-\gamma \mathcal{G}\left(\Theta_{n}\right)$ and $\gamma \leq\left(4 V\left(\Theta_{0}\right)+2\right)^{-1}$. Then

(i) it holds that $\sup _{n \in \mathbb{N}_{0}}\left\|\Theta_{n}\right\| \leq\left[V\left(\Theta_{0}\right)\right]^{1 / 2}<\infty$ and

(ii) it holds that $\lim \sup _{n \rightarrow \infty} \mathcal{L}_{\infty}\left(\Theta_{n}\right)=0$.

Proof of Theorem 4.4. Observe that Lemma 4.3 proves that for all $n \in \mathbb{N}_{0}$ we have that $V\left(\Theta_{n}\right) \leq V\left(\Theta_{n-1}\right) \leq \cdots \leq V\left(\Theta_{0}\right)$. This and the fact that $\forall n \in \mathbb{N}_{0}:\left\|\Theta_{n}\right\| \leq\left[V\left(\Theta_{n}\right)\right]^{1 / 2}$ establish item (i). Next note that Lemma 4.3 implies for all $N \in \mathbb{N}$ that

$$
\sum_{n=0}^{N-1}\left(4 \gamma \mathcal{L}_{\infty}\left(\Theta_{n}\right)\right) \leq \sum_{n=0}^{N-1}\left(V\left(\Theta_{n}\right)-V\left(\Theta_{n+1}\right)\right)=V\left(\Theta_{0}\right)-V\left(\Theta_{N}\right) \leq V\left(\Theta_{0}\right) .
$$

Hence, we have that

$$
\sum_{n=0}^{\infty} \mathcal{L}_{\infty}\left(\Theta_{n}\right) \leq \frac{V\left(\Theta_{0}\right)}{4 \gamma}<\infty
$$

This shows that $\limsup _{n \rightarrow \infty} \mathcal{L}_{\infty}\left(\Theta_{n}\right)=0$. The proof of Theorem 4.4 is thus complete.

Corollary 4.5. Assume Setting 2.1, let $\gamma \in(0, \infty)$, and let $\Theta=\left(\Theta_{n}\right)_{n \in \mathbb{N}_{0}}: \mathbb{N}_{0} \rightarrow \mathbb{R}^{3 H+1}$ satisfy for all $n \in \mathbb{N}_{0}$ that $\Theta_{n+1}=\Theta_{n}-\gamma \mathcal{G}\left(\Theta_{n}\right)$ and $\gamma \leq\left[12\left\|\Theta_{0}\right\|^{2}+32 \alpha^{2}+2\right]^{-1}$. Then

(i) it holds that $\sup _{n \in \mathbb{N}_{0}}\left\|\Theta_{n}\right\| \leq\left[V\left(\Theta_{0}\right)\right]^{1 / 2}<\infty$ and

(ii) it holds that $\lim _{\sup } \operatorname{su}_{n \rightarrow \infty} \mathcal{L}_{\infty}\left(\Theta_{n}\right)=0$.

Proof of Corollary 4.5. Observe that Proposition 2.12 proves that $4 V\left(\Theta_{0}\right)+2 \leq 12\left\|\Theta_{0}\right\|^{2}+$ $32 \alpha^{2}+2$. Hence, we have that $\gamma \leq\left(4 V\left(\Theta_{0}\right)+2\right)^{-1}$. Combining this with Theorem 4.4 completes the proof of Corollary 4.5.

\subsection{Gradient descent processes with random initializations}

Corollary 4.6. Assume Setting 2.1, let $c, \gamma \in(0, \infty)$, let $(\Omega, \mathcal{F}, \mathbb{P})$ be a probability space, let $\Theta=\left(\Theta_{n}\right)_{n \in \mathbb{N}_{0}}: \Omega \times \mathbb{N}_{0} \rightarrow \mathbb{R}^{3 H+1}$ be a stochastic process, assume $\Theta_{0}(\Omega) \subseteq[-c, c]^{3 H+1}$, assume for all $n \in \mathbb{N}_{0}$ that $\Theta_{n+1}=\Theta_{n}-\gamma \mathcal{G}\left(\Theta_{n}\right)$, and assume $\gamma \leq\left[12 c^{2}(3 H+1)+32 \alpha^{2}+2\right]^{-1}$. Then

(i) it holds that $\sup _{\omega \in \Omega} \sup _{n \in \mathbb{N}_{0}}\left\|\Theta_{n}(\omega)\right\| \leq\left[3 c^{2}(3 H+1)+8 \alpha^{2}\right]^{1 / 2}<\infty$,

(ii) it holds for all $\omega \in \Omega$ that $\lim \sup _{n \rightarrow \infty} \mathcal{L}_{\infty}\left(\Theta_{n}(\omega)\right)=0$, and 
(iii) it holds that $\lim \sup _{n \rightarrow \infty} \mathbb{E}\left[\mathcal{L}_{\infty}\left(\Theta_{n}\right)\right]=0$.

Proof of Corollary 4.6. Note that Proposition 2.12 demonstrates for all $\phi \in[-c, c]^{3 H+1}$ that

$$
V(\phi) \leq 3\|\phi\|^{2}+8 \alpha^{2} \leq 3 c^{2}(3 H+1)+8 \alpha^{2} .
$$

Hence, we have for all $\phi \in[-c, c]^{3 H+1}$ that

$$
\gamma \leq\left[12 c^{2}(3 H+1)+32 \alpha^{2}+2\right]^{-1} \leq[4 V(\phi)+2]^{-1} .
$$

This demonstrates for all $\omega \in \Omega$ that $\gamma \leq\left(4 V\left(\Theta_{0}(\omega)\right)+2\right)^{-1}$. Lemma 4.3 and (78) hence prove that for all $\omega \in \Omega, n \in \mathbb{N}_{0}$ we have that $\left\|\Theta_{n}(\omega)\right\| \leq\left[V\left(\Theta_{n}(\omega)\right)\right]^{1 / 2} \leq\left[V\left(\Theta_{0}(\omega)\right)\right]^{1 / 2} \leq$ $\left[3 c^{2}(3 H+1)+8 \alpha^{2}\right]^{1 / 2}$. This establishes item (i). Next observe that Theorem 4.4 shows for all $\omega \in \Omega$ that $\lim \sup _{n \rightarrow \infty} \mathcal{L}_{\infty}\left(\Theta_{n}(\omega)\right)=0$, which proves item (ii). Furthermore, note that Lemma 4.3 assures that for all $\omega \in \Omega, N \in \mathbb{N}$ it holds that

$$
\sum_{n=0}^{N-1}\left(4 \gamma \mathcal{L}_{\infty}\left(\Theta_{n}(\omega)\right)\right) \leq \sum_{n=0}^{N-1}\left(V\left(\Theta_{n+1}(\omega)\right)-V\left(\Theta_{n}(\omega)\right)\right) \leq V\left(\Theta_{0}(\omega)\right) .
$$

Proposition 2.12 hence shows that for all $\omega \in \Omega$ we have that

$$
\sum_{n=0}^{\infty} \mathcal{L}_{\infty}\left(\Theta_{n}(\omega)\right) \leq \frac{V\left(\Theta_{0}(\omega)\right)}{4 \gamma} \leq \frac{3\left\|\Theta_{0}(\omega)\right\|^{2}+8 \alpha^{2}}{4 \gamma}
$$

Combining this, item (ii), and the dominated convergence theorem establishes item (iii). The proof of Corollary 4.6 is thus complete.

\section{A priori estimates for general target functions}

The key ingredient in our convergence proofs for gradient flow and GD processes in Sections 3 and 4 are suitable a priori estimates for the gradient flow processes (see Lemma 3.2 in Subsection 3.1) and the GD processes (see Lemma 4.3 in Subsection 4.1). To initiate further research activities of this kind, we derive in this section related a priori bounds in the case of general target functions. For details we refer to (83) and (84) in Proposition 5.1 below.

Proposition 5.1. Let $H \in \mathbb{N}, f \in C([0,1], \mathbb{R})$, let $\mathfrak{w}=\left(\left(\mathfrak{w}_{1}^{\phi}, \ldots, \mathfrak{w}_{H}^{\phi}\right)\right)_{\phi \in \mathbb{R}^{3 H+1}}: \mathbb{R}^{3 H+1} \rightarrow$ $\mathbb{R}^{H}, \mathfrak{b}=\left(\left(\mathfrak{b}_{1}^{\phi}, \ldots, \mathfrak{b}_{H}^{\phi}\right)\right)_{\phi \in \mathbb{R}^{3 H+1}}: \mathbb{R}^{3 H+1} \rightarrow \mathbb{R}^{H}, \mathfrak{v}=\left(\left(\mathfrak{v}_{1}^{\phi}, \ldots, \mathfrak{v}_{H}^{\phi}\right)\right)_{\phi \in \mathbb{R}^{3 H+1}}: \mathbb{R}^{3 H+1} \rightarrow \mathbb{R}^{H}$, $\mathfrak{c}=\left(\mathfrak{c}^{\phi}\right)_{\phi \in \mathbb{R}^{3 H+1}}: \mathbb{R}^{3 H+1} \rightarrow \mathbb{R}$, and $\|\cdot\|: \mathbb{R}^{3 H+1} \rightarrow[0, \infty)$ satisfy for all $\phi=\left(\phi_{1}, \ldots, \phi_{3 H+1}\right) \in$ $\mathbb{R}^{3 H+1}, j \in\{1,2, \ldots, H\}$ that $\mathfrak{w}_{j}^{\phi}=\phi_{j}, \mathfrak{b}_{j}^{\phi}=\phi_{H+j}, \mathfrak{v}_{j}^{\phi}=\phi_{2 H+j}, \mathfrak{c}^{\phi}=\phi_{3 H+1}$, and $\|\phi\|=$ $\left[\sum_{i=1}^{3 H+1}\left|\phi_{i}\right|^{2}\right]^{1 / 2}$, let $\mathcal{N}=\left(\mathcal{N}^{\phi}\right)_{\phi \in \mathbb{R}^{3 H+1}}: \mathbb{R}^{3 H+1} \rightarrow C(\mathbb{R}, \mathbb{R})$ and $\mathcal{L}: \mathbb{R}^{3 H+1} \rightarrow \mathbb{R}$ satisfy for all $\phi \in \mathbb{R}^{3 H+1}, x \in \mathbb{R}$ that $\mathscr{N}^{\phi}(x)=\mathfrak{c}^{\phi}+\sum_{j=1}^{H} \mathfrak{v}_{j}^{\phi} \max \left\{\mathfrak{w}_{j}^{\phi} x+\mathfrak{b}_{j}^{\phi}, 0\right\}$ and $\mathcal{L}(\phi)=\int_{0}^{1}\left(\mathcal{N}^{\phi}(y)-\right.$ $f(y))^{2} \mathrm{~d} y$, let $V: \mathbb{R}^{3 H+1} \rightarrow \mathbb{R}$ and $\mathcal{G}=\left(\mathcal{G}_{1}, \ldots, \mathcal{G}_{3 H+1}\right): \mathbb{R}^{3 H+1} \rightarrow \mathbb{R}^{3 H+1}$ satisfy for all $\phi \in$ $\mathbb{R}^{3 H+1}, j \in\{1,2, \ldots, H\}$ that $V(\phi)=\|\phi\|^{2}+\left|\mathfrak{c}^{\phi}\right|^{2}$ and

$$
\begin{aligned}
\mathcal{G}_{j}(\phi) & =2 \mathfrak{v}_{j}^{\phi} \int_{0}^{1} x\left(\mathcal{N}^{\phi}(x)-f(x)\right) \mathbb{1}_{(0, \infty)}\left(\mathfrak{w}_{j}^{\phi} x+\mathfrak{b}_{j}^{\phi}\right) \mathrm{d} x, \\
\mathcal{G}_{H+j}(\phi) & =2 \mathfrak{v}_{j}^{\phi} \int_{0}^{1}\left(\mathcal{N}^{\phi}(x)-f(x)\right) \mathbb{1}_{(0, \infty)}\left(\mathfrak{w}_{j}^{\phi} x+\mathfrak{b}_{j}^{\phi}\right) \mathrm{d} x, \\
\mathcal{G}_{2 H+j}(\phi) & =2 \int_{0}^{1}\left[\max \left\{\mathfrak{w}_{j}^{\phi} x+\mathfrak{b}_{j}^{\phi}, 0\right\}\right]\left(\mathcal{N}^{\phi}(x)-f(x)\right) \mathrm{d} x, \\
\mathcal{G}_{3 H+1}(\phi) & =2 \int_{0}^{1}\left(\mathcal{N}^{\phi}(x)-f(x)\right) \mathrm{d} x,
\end{aligned}
$$

and let $\Theta \in C\left([0, \infty), \mathbb{R}^{3 H+1}\right)$ satisfy for all $t \in[0, \infty)$ that $\Theta_{t}=\Theta_{0}-\int_{0}^{t} \mathcal{G}\left(\Theta_{s}\right) \mathrm{d}$ s. Then 
(i) it holds for all $t \in[0, \infty)$ that

$$
V\left(\Theta_{t}\right)=V\left(\Theta_{0}\right)-8 \int_{0}^{t} \int_{0}^{1} \mathcal{N}^{\Theta_{s}}(x)\left(\mathcal{N}^{\Theta_{s}}(x)-f(x)\right) \mathrm{d} x \mathrm{~d} s \leq V\left(\Theta_{0}\right)+2 t \int_{0}^{1}|f(x)|^{2} \mathrm{~d} x
$$

and

(ii) it holds for all $t \in[0, \infty)$ that

$$
\left\|\Theta_{t}\right\| \leq\left(V\left(\Theta_{0}\right)\right)^{1 / 2}+\left[2 \int_{0}^{1}|f(x)|^{2} \mathrm{~d} x\right]^{1 / 2} t^{1 / 2}
$$

Proof of Proposition 5.1. Throughout this proof let $\langle\cdot, \cdot\rangle: \mathbb{R}^{3 H+1} \times \mathbb{R}^{3 H+1} \rightarrow \mathbb{R}$ satisfy for all $\phi=\left(\phi_{1}, \ldots, \phi_{3 H+1}\right), \psi=\left(\psi_{1}, \ldots, \psi_{3 H+1}\right) \in \mathbb{R}^{3 H+1}$ that $\langle\phi, \psi\rangle=\sum_{i=1}^{3 H+1} \phi_{i} \psi_{i}$. Observe that for all $\phi \in \mathbb{R}^{3 H+1}$ it holds that

$$
(\nabla V)(\phi)=2\left(\mathfrak{w}_{1}^{\phi}, \mathfrak{w}_{2}^{\phi}, \ldots, \mathfrak{w}_{H}^{\phi}, \mathfrak{b}_{1}^{\phi}, \mathfrak{b}_{2}^{\phi}, \ldots, \mathfrak{b}_{H}^{\phi}, \mathfrak{v}_{1}^{\phi}, \mathfrak{v}_{2}^{\phi}, \ldots, \mathfrak{v}_{H}^{\phi}, 2 \mathfrak{c}^{\phi}\right) .
$$

This implies for all $\phi \in \mathbb{R}^{3 H+1}$ that

$$
\begin{aligned}
& \langle(\nabla V)(\phi), \mathcal{G}(\phi)\rangle \\
& =4\left[\sum_{j=1}^{H} \mathfrak{v}_{j}^{\phi} \int_{0}^{1}\left[\max \left\{\mathfrak{w}_{j}^{\phi} x+\mathfrak{b}_{j}^{\phi}, 0\right\}\right]\left(\mathcal{N}^{\phi}(x)-f(x)\right) \mathrm{d} x\right]+8 \mathfrak{c}^{\phi}\left[\int_{0}^{1}\left(\mathcal{N}^{\phi}(x)-f(x)\right) \mathrm{d} x\right] \\
& +4\left[\sum_{j=1}^{H} \mathfrak{v}_{j}^{\phi} \int_{0}^{1}\left(\mathfrak{w}_{j}^{\phi} x+\mathfrak{b}_{j}^{\phi}\right)\left(\mathcal{N}^{\phi}(x)-f(x)\right) \mathbb{1}_{(0, \infty)}\left(\mathfrak{w}_{j}^{\phi} x+\mathfrak{b}_{j}^{\phi}\right) \mathrm{d} x\right] \\
& =8\left[\int_{0}^{1}\left(\sum_{j=1}^{H} \mathfrak{v}_{j}^{\phi}\left[\max \left\{\mathfrak{w}_{j}^{\phi} x+\mathfrak{b}_{j}^{\phi}, 0\right\}\right]\right)\left(\mathcal{N}^{\phi}(x)-f(x)\right) \mathrm{d} x\right]+8 \mathfrak{c}^{\phi}\left[\int_{0}^{1}\left(\mathcal{N}^{\phi}(x)-f(x)\right) \mathrm{d} x\right] \\
& =8 \int_{0}^{1} \mathscr{N}^{\phi}(x)\left(\mathcal{N}^{\phi}(x)-f(x)\right) \mathrm{d} x .
\end{aligned}
$$

Next note that the fact that for all $x, y \in \mathbb{R}$ it holds that $x(x-y)=\left(x-\frac{y}{2}\right)^{2}-\frac{1}{4} y^{2} \geq-\frac{1}{4} y^{2}$ ensures that for all $x \in[0,1], \phi \in \mathbb{R}^{3 H+1}$ it holds that $\mathscr{N}^{\phi}(x)\left(\mathcal{N}^{\phi}(x)-f(x)\right) \geq-\frac{1}{4}(f(x))^{2}$. Hence, we have for all $\phi \in \mathbb{R}^{3 H+1}$ that

$$
\langle(\nabla V)(\phi), \mathcal{G}(\phi)\rangle \geq-2 \int_{0}^{1}|f(x)|^{2} \mathrm{~d} x
$$

This, (86), the fact that $V \in C^{\infty}\left(\mathbb{R}^{3 H+1}, \mathbb{R}\right)$, and Lemma 3.1 shows for all $t \in[0, \infty)$ that

$$
\begin{aligned}
V\left(\Theta_{t}\right)-V\left(\Theta_{0}\right) & =-\int_{0}^{t}\left\langle(\nabla V)\left(\Theta_{s}\right), \mathcal{G}\left(\Theta_{s}\right)\right\rangle \mathrm{d} s \\
& =-8 \int_{0}^{t} \int_{0}^{1} \mathcal{N}^{\Theta_{s}}(x)\left(\mathcal{N}^{\Theta_{s}}(x)-f(x)\right) \mathrm{d} x \mathrm{~d} s \\
& \leq 2 \int_{0}^{t} \int_{0}^{1}|f(x)|^{2} \mathrm{~d} x \mathrm{~d} s=2 t \int_{0}^{1}|f(x)|^{2} \mathrm{~d} x .
\end{aligned}
$$

This proves item (i). Next observe that item (i) and the fact that $\forall \phi \in \mathbb{R}^{3 H+1}:\|\phi\|^{2} \leq V(\phi)$ demonstrate that for all $t \in[0, \infty)$ it holds that

$$
\left\|\Theta_{t}\right\| \leq\left(V\left(\Theta_{t}\right)\right)^{1 / 2} \leq\left[V\left(\Theta_{0}\right)+2 t \int_{0}^{1}|f(x)|^{2} \mathrm{~d} x\right]^{1 / 2} .
$$

Combining this and the fact that $\forall x, y \in[0, \infty):(x+y)^{1 / 2} \leq x^{1 / 2}+y^{1 / 2}$ ensures that for all $t \in[0, \infty)$ we have that

$$
\left\|\Theta_{t}\right\| \leq\left(V\left(\Theta_{0}\right)\right)^{1 / 2}+\left[2 \int_{0}^{1}|f(x)|^{2} \mathrm{~d} x\right]^{1 / 2} t^{1 / 2}
$$

This establishes item (ii). The proof of Proposition 5.1 is thus complete. 


\section{Acknowledgments}

Benno Kuckuck is gratefully acknowledged for several helpful suggestions. This work has been funded by the Deutsche Forschungsgemeinschaft (DFG, German Research Foundation) under Germany's Excellence Strategy EXC 2044-390685587, Mathematics Münster: DynamicsGeometry-Structure.

\section{References}

[1] Akyildiz, Ö. D., And Sabanis, S. Nonasymptotic analysis of Stochastic Gradient Hamiltonian Monte Carlo under local conditions for nonconvex optimization. arXiv:2002.05465 (2021), 26 pages.

[2] Allen-Zhu, Z., Li, Y., And Liang, Y. Learning and generalization in overparameterized neural networks, going beyond two layers. In Advances in Neural Information Processing Systems (2019), H. Wallach, H. Larochelle, A. Beygelzimer, F. d'Alché-Buc, E. Fox, and R. Garnett, Eds., vol. 32, Curran Associates, Inc., pp. 6158-6169.

[3] Allen-Zhu, Z., Li, Y., And Song, Z. A convergence theory for deep learning via overparameterization. In Proceedings of the 36th International Conference on Machine Learning (09-15 Jun 2019), K. Chaudhuri and R. Salakhutdinov, Eds., vol. 97 of Proceedings of Machine Learning Research, PMLR, pp. 242-252.

[4] BACH, F. Breaking the curse of dimensionality with convex neural networks. Journal of Machine Learning Research 18, 19 (2017), 1-53.

[5] Bach, F., And Moulines, E. Non-strongly-convex smooth stochastic approximation with convergence rate $O(1 / n)$. In Advances in Neural Information Processing Systems (2013), C. J. C. Burges, L. Bottou, M. Welling, Z. Ghahramani, and K. Q. Weinberger, Eds., vol. 26, Curran Associates, Inc., pp. 773-781.

[6] Cheridito, P., Jentzen, A., And Rossmannek, F. Non-convergence of stochastic gradient descent in the training of deep neural networks. Journal of Complexity (2020), 101540.

[7] Chizat, L., And Bach, F. On the global convergence of gradient descent for overparameterized models using optimal transport. In Advances in Neural Information Processing Systems (2018), S. Bengio, H. Wallach, H. Larochelle, K. Grauman, N. Cesa-Bianchi, and R. Garnett, Eds., vol. 31, Curran Associates, Inc., pp. 3036-3046.

[8] Du, S., Lee, J., Li, H., Wang, L., And Zhai, X. Gradient descent finds global minima of deep neural networks. In Proceedings of the 36th International Conference on Machine Learning (Long Beach, California, USA, 6 2019), K. Chaudhuri and R. Salakhutdinov, Eds., vol. 97 of Proceedings of Machine Learning Research, PMLR, pp. 1675-1685.

[9] Du, S. S., Zhai, X., Poczós, B., And Singh, A. Gradient descent provably optimizes over-parameterized neural networks. arXiv:1810.02054 (2018), 19 pages.

[10] E, W., MA, C., AND Wu, L. A comparative analysis of optimization and generalization properties of two-layer neural network and random feature models under gradient descent dynamics. Science China Mathematics 63, 7 (2020), 1235-1258.

[11] Fehrman, B., Gess, B., And Jentzen, A. Convergence rates for the stochastic gradient descent method for non-convex objective functions. Journal of Machine Learning Research 21, 136 (2020), 1-48. 
[12] Hanin, B. Which neural net architectures give rise to exploding and vanishing gradients? In Advances in Neural Information Processing Systems (2018), S. Bengio, H. Wallach, H. Larochelle, K. Grauman, N. Cesa-Bianchi, and R. Garnett, Eds., vol. 31, Curran Associates, Inc., pp. 582-591.

[13] Hanin, B., And Rolnick, D. How to start training: The effect of initialization and architecture. In Advances in Neural Information Processing Systems (2018), S. Bengio, H. Wallach, H. Larochelle, K. Grauman, N. Cesa-Bianchi, and R. Garnett, Eds., vol. 31, Curran Associates, Inc., pp. 571-581.

[14] Jacot, A., Gabriel, F., And Hongler, C. Neural tangent kernel: Convergence and generalization in neural networks. In Advances in Neural Information Processing Systems (2018), S. Bengio, H. Wallach, H. Larochelle, K. Grauman, N. Cesa-Bianchi, and R. Garnett, Eds., vol. 31, Curran Associates, Inc., pp. 8571-8580.

[15] Jentzen, A., Kuckuck, B., Neufeld, A., And von Wurstemberger, P. Strong error analysis for stochastic gradient descent optimization algorithms. IMA Journal of Numerical Analysis 41, 1 (2021), 455-492.

[16] Jentzen, A., And von Wurstemberger, P. Lower error bounds for the stochastic gradient descent optimization algorithm: Sharp convergence rates for slowly and fast decaying learning rates. Journal of Complexity 57 (2020), 101438.

[17] Lei, Y., Hu, T., Li, G., And Tang, K. Stochastic gradient descent for nonconvex learning without bounded gradient assumptions. IEEE Transactions on Neural Networks and Learning Systems 31, 10 (2020), 4394-4400.

[18] Li, Y., AND LiANG, Y. Learning overparameterized neural networks via stochastic gradient descent on structured data. In Advances in Neural Information Processing Systems (2018), S. Bengio, H. Wallach, H. Larochelle, K. Grauman, N. Cesa-Bianchi, and R. Garnett, Eds., vol. 31, Curran Associates, Inc., pp. 8157-8166.

[19] Lovas, A., Lytras, I., RÁsonyi, M., And Sabanis, S. Taming neural networks with TUSLA: Non-convex learning via adaptive stochastic gradient Langevin algorithms. arXiv:2006.14514 (2020), 29 pages.

[20] Lu, L., Shin, Y., Su, Y., and Karniadakis, G. E. Dying ReLU and initialization: Theory and numerical examples. Communications in Computational Physics 28, 5 (2020), 1671-1706.

[21] Moulines, E., And BaCh, F. Non-asymptotic analysis of stochastic approximation algorithms for machine learning. In Advances in Neural Information Processing Systems (2011), J. Shawe-Taylor, R. Zemel, P. Bartlett, F. Pereira, and K. Q. Weinberger, Eds., vol. 24, Curran Associates, Inc., pp. 451-459.

[22] RUder, S. An overview of gradient descent optimization algorithms. arXiv:1609.04747 (2017), 14 pages.

[23] Sankararaman, K. A., De, S., Xu, Z., Huang, W. R., and Goldstein, T. The impact of neural network overparameterization on gradient confusion and stochastic gradient descent. arXiv:1904.06963 (2020), 28 pages.

[24] Shin, Y., And Karniadakis, G. E. Trainability of ReLU networks and data-dependent initialization. Journal of Machine Learning for Modeling and Computing 1, 1 (2020), 39-74. 
[25] Wu, L., MA, C., ANd E, W. How SGD selects the global minima in over-parameterized learning: A dynamical stability perspective. In Advances in Neural Information Processing Systems (2018), S. Bengio, H. Wallach, H. Larochelle, K. Grauman, N. Cesa-Bianchi, and R. Garnett, Eds., vol. 31, Curran Associates, Inc., pp. 8279-8288.

[26] Zou, D., Cao, Y., Zhou, D., And Gu, Q. Gradient descent optimizes over-parameterized deep ReLU networks. Machine Learning 109 (2020), 467-492. 